\title{
THE DIFFERENTIAL EQUATION OF THE ELLIPTIC CYLINDER*
}

\author{
BY \\ J. H. MCDONALD
}

1. The differential equation of the elliptic cylinder, often called Mathieu's equation, may be written in either of the forms

or

$$
\frac{d^{2} y}{d z^{2}}+\left(a+k^{2} \cos ^{2} z\right) y=0
$$

$$
\frac{d^{2} y}{d z^{2}}+\left(a+k^{2} \cosh ^{2} z\right) y=0 .
$$

It is not capable of reference to the hypergeometric type and so requires methods peculiar to itself. The principal difficulty in the solution of the equation consists in the determination of a number, the characteristic exponent, which appears in the solution. It is known from general considerations of the theory of linear differential equations that for the trigonometric form of the equation, $y$ may be expressed as

$$
c_{1} e^{2 \mu i z} \phi\left(e^{2 i z}\right)+c_{2} e^{-2 \mu i z} \psi\left(e^{2 i z}\right),
$$

the parameters $a$ and $k^{2}$ being supposed arbitrary. The functions $\phi\left(e^{2 i s}\right)$, $\psi\left(e^{2 i z}\right)$ are series of positive and negative integral powers of $e^{2 i z}$, and the quantity $\mu$ which is a function of $a$ and $k^{2}$ is the exponent in question, and its determination necessitates extended developments. If $\mu$ has been found the coefficients of the series for $\phi\left(e^{2 i z}\right), \psi\left(e^{2 i z}\right)$ must be investigated. They satisfy a particular difference equation which is not of a simple type.

Of special importance are those cases of the equation for which there exists a periodic solution with period $\pi$ or $2 \pi$. These solutions which are sometimes called Mathieu's functions do not exist in the general case, but require that $a$ should have special values when $k^{2}$ is given. The determination of these values is an important problem. It is to be noticed that in these cases the general solution referred to above is no longer valid. $\dagger$ Periodic solutions present themselves in problems of mathematical physics, for example in

\footnotetext{
* Presented to the Society, San Francisco Section, April 4, 1925; received by the editors in August, 1926.

† H. Weber, Die Partiellen Differential-Gleichungen der Mathematischen Physik, vol. 2, p. 265. Bruns, Astronomische Nachrichten, No. 2533, p. 193, No. 2553, p. 129.
} 
determining the vibrations of an elliptic membrane, while the solutions which are not periodic find an application in the theory of orbits.

The solution of the problems indicated in this summary has previously been attempted without complete success. For the bibliography reference may be had to Whittaker and Watson, Modern Analysis, 3d edition, chapter xix. The most valuable references are to papers by Dougall (The solution of Mathieu's differential equation, Proceedings of the Edinburgh Mathematical Society, vol. 34, part 1, p. 176, 1915-16) and Whittaker (On the functions associated with the elliptic cylinder in harmonic analysis, Proceedings of the Fifth International Congress of Mathematicians, 1912, Cambridge). Dougall obtains, among other results, an equation for the characteristic exponent but not in an explicit form. Whittaker proves that the periodic solutions satisfy an integral equation which he uses to derive the expressions for some of the earlier cases.

In the present paper the problems stated above are treated more fully than has previously been done. First the general case is considered. The difference equation for the coefficients of the solution of the differential equation is replaced by an infinite system of equations, and their solution given in two different forms. The characteristic exponent $\mu$ is shown to satisfy an equation found in two corresponding forms.

Second, the characteristic exponent is studied in detail. The differential equation is replaced by a Riccati equation, a particular solution of which is found, and from this solution an explicit expression for $\mu$ is determined. This expression which is valid only within a limited range of values of $k^{2}$ leads to a form of the equation for $\mu$ valid for all values of $k^{2}$. It is found that $\mu$ is an analytic function of $k^{2}$ when $a$ is given, and the character of the singular points of $\mu$ is determined. The convergence of the solution constructed in the first part of the paper is then proved.

In the third place periodic solutions are considered. On the supposition that they exist they are determined in two different forms. Proof is then given of the existence of periodic solutions and a determination of all of them is made. An equation for the parameter $a$ is given to each of whose roots a periodic solution belongs. This equation has no multiple roots if $k^{2}$ is real. Two expressions for periodic solutions are given in forms valid for all values of $k^{2}$. The parameters $a$ and the solutions are analytic functions of $k^{2}$.

Finally some miscellaneous results are collected. The differential equation is shown not to have two periodic solutions for the same values of $a$ and $k^{2}$, and if $k^{2}$ is real it is shown that each $a$ is a decreasing function of $k^{2}$. Some indications as to the determination of the singular points of $a$ are added. 
The general CASE

2. The equation of the elliptic cylinder may be written in the form

$$
d^{2} y / d z^{2}+\left(\frac{1}{2} k^{2} c^{2} \cosh 2 z-s^{2}\right) y=0 .
$$

Then a solution*

$$
y=\sum_{-\infty}^{+\infty} c_{n} e^{(2 n+\omega) z}
$$

exists where $\omega$ and the coefficients $c_{n}$ are independent of $z$. Substitution of the solution in the equation gives

$$
\left\{(2 n+\omega)^{2}-s^{2}\right\} c_{n}+\frac{1}{4} k^{2} c^{2}\left(c_{n-1}+c_{n+1}\right)=0 .
$$

If $c_{n}$ is replaced by $(-1)^{n} C_{n}$,

$$
C_{n+1}+C_{n-1}=\frac{\left(n+\frac{1}{2} \omega\right)^{2}-\frac{1}{4} s^{2}}{\frac{1}{16} k^{2} c^{2}} C_{n} .
$$

Put $\frac{1}{3} \omega=\mu, \frac{1}{2} s=r, \frac{1}{4} k c=\lambda$, and this equation becomes

Let

$$
C_{n+1}+C_{n-1}=\frac{(n+\mu)^{2}-r^{2}}{\lambda^{2}} C_{n} .
$$

$$
-\frac{\lambda^{2}}{(n+\mu)^{2}-r^{2}}=x_{n} ;
$$

then if $n$ varies from $-\infty$ to $+\infty$ there results the system of equations

$$
\begin{aligned}
x_{0} C_{-1}+C_{0}+x_{0} C_{1} & =0, \\
x_{1} C_{0}+C_{1}+ & x_{1} C_{2}=0, \\
x_{2} C_{1}+ & C_{2}+x_{2} C_{3}=0, \\
& x_{3} C_{2}+C_{3}+x_{3} C_{4}=0,
\end{aligned}
$$

Any solution of this system of equations which makes $\sum_{-\infty}^{+\infty} c_{n} e^{(2 n+\omega) s}$ convergent furnishes a solution of the differential equation.

3. If it is supposed that $(n+\mu)^{2}-r^{2} \neq 0$ for every value of $n$, then the infinite determinant

- Floquet, Annales de l'Ecole Normale Supérieure, 1883. 


$$
\left|\begin{array}{llllll}
1 & x_{i} & 0 & 0 & 0 & \cdots \\
x_{i+1} & 1 & x_{i+1} & 0 & 0 & \ldots \\
0 & x_{i+2} & 1 & x_{i+2} & 0 & \ldots \\
. & . & . & . & . & \ldots
\end{array}\right|=\left(x_{i}\right)
$$

is convergent for all values of $i$. This is true since a sufficient condition of convergence of the infinite determinant

is that the series

$$
\left|\begin{array}{cccc}
1+c_{11} & c_{12} & c_{13} & \cdots \\
c_{21} & 1+c_{22} & c_{28} & \cdots \\
\cdot & \cdot & \cdot & \cdots
\end{array}\right|
$$

$$
c_{11}+c_{12}+c_{21}+c_{13}+c_{22}+c_{31}+\cdots
$$

be absolutely convergent. ${ }^{*}$ This condition for the determinant $\left(x_{i}\right)$ is that

$$
\frac{\lambda^{2}}{(i+\mu)^{2}-r^{2}}+2 \frac{\lambda^{2}}{(i+1+\mu)^{2}-r^{2}}+2 \frac{\lambda^{2}}{(i+2+\mu)^{2}-r^{2}}+\cdots
$$

be absolutely convergent. Since the terms of this series are comparable in absolute value with those of the series

$$
\frac{1}{i^{2}}+\frac{1}{(i+1)^{2}}+\frac{1}{(i+2)^{2}}+\cdots
$$

the condition is satisfied. By expanding in terms of the elements of the first row it may be verified that the determinants $\left(x_{i}\right)$ satisfy the recurrence relation

$$
\left(x_{i}\right)=\left(x_{i+1}\right)-x_{i} x_{i+1}\left(x_{i+2}\right) .
$$

To examine the character of the expansion of $\left(x_{i}\right)$, consider the particular case $\left(x_{1}\right)$ and let

$$
D_{n}=\left|\begin{array}{cccccccc}
1 & x_{1} & 0 & 0 & . & . & . & . \\
x_{2} & 1 & x_{2} & 0 & . & . & . & . \\
. & . & . & . & . & . & . & . \\
0 & 0 & 0 & 0 & . & . & x_{n} & 1
\end{array}\right|
$$

be the determinant of the first $n$ rows and columns of $\left(x_{1}\right)$. Then if $D_{n}$ is expanded in terms of the last row,

$$
D_{n}=D_{n-1}-x_{n-1} x_{n} D_{n-2} \text {. }
$$

* Kowalewski, Einfulhrung in die Determinantentheorie, p. 372. 
From this recurrence formula it is seen that the terms of the expansion of $D_{n}$ which contain $x_{n}$ consist of the product $x_{n-1} x_{n}$ multiplied by $-D_{n-2}$, and those terms which do not contain $x_{n}$ are the terms of $D_{n-1}$. Now consider the sum

$$
S_{n}=1-\sum_{i} x_{i} x_{i+1}+\sum_{i>i+1} x_{i} x_{i+1} x_{j} x_{i+1}-\sum_{k>j+1, i>i+1} x_{i} x_{i+1} x_{j} x_{i+1} x_{k} x_{k+1}+\cdots
$$

Let the subscripts, subject to the inequalities, take the values from 1 to $n$ in every possible way, and let the series be continued as long as it is possible to construct terms in which no subscript is greater than $n$. Then the terms of $S_{n}$ which contain $x_{n}$ consist of the product $x_{n-1} x_{n}$ multiplied by $-S_{n-2}$, and those which do not contain $x_{n}$ are the terms of $S_{n-1}$. Moreover $S_{1}=D_{1}$, $S_{2}=D_{2}$, etc. Therefore $S_{n}=D_{n}$.

The determinant $\left(x_{1}\right)$ which is infinite has therefore the formal expansion

$$
1-\sum_{i} x_{i} x_{i+1}+\sum_{i>i+1} x_{i} x_{i+1} x_{i} x_{i+1}-\cdots
$$

where the numbers $i, j, \cdots$ are any succession taken from $1,2, \cdots$ subject to the inequalities. Since this series is convergent it is an integral function of $\lambda$ of the form

$$
1+a_{4} \lambda^{4}+a_{8} \lambda^{8}+\cdots \cdot
$$

Though not relevant to the subject it may be noticed that the number of terms of a given order in $D_{n}$ is figurate. If $f_{n, r}$ denotes the $n$th figurate number of the rth order, the number of terms of order $2 p$ in $D_{2 n}$ is $f_{2 n+1-2 p, p+1}$ and in $D_{2 n+1}$ is $f_{2 n+2-2 p, p+1}, 0<p \leqq n$. These are included in the statement that in $D_{n}$ the number of terms of order $2 p$ is $f_{n+1-2 p, p+1}, 0<2 p \leqq n$.

4. A solution of the system of equations for $C_{i}(\S 2)$ such that $C_{n} \rightarrow 0$ as $n \rightarrow \infty$ can now be obtained. Consider the first $n$ equations for which $i \geqq 0$ :

$$
\begin{aligned}
& x_{1} C_{0}+C_{1}+x_{1} C_{2}=0, \\
& x_{2} C_{1}+C_{2}+x_{2} C_{3}=0, \\
& \cdot \cdot \cdot \cdot \cdot \cdot \cdot \cdot \\
& x_{n} C_{n-1}+C_{n}+x_{n} C_{n+1}=0 .
\end{aligned}
$$

If it be assumed that $C_{i} \neq 0, i \geqq 0, C_{0}$ may be put equal to unity. Then the value of $C_{1}$ is given by

$$
\left|\begin{array}{cccccc}
-x_{1} & x_{1} & 0 & \cdot & \cdot & \cdot \\
0 & 1 & x_{2} & \cdot & \cdot & \cdot \\
\cdot & \cdot & \cdot & \cdot & \cdot & \cdot \\
-x_{n} C_{n+1} & 0 & 0 & \cdot & x_{n} & 1
\end{array}\right| \div\left|\begin{array}{ccccccc}
1 & x_{1} & 0 & 0 & \cdot & \cdot & \cdot \\
x_{2} & 1 & x_{2} & 0 & \cdot & \cdot & \cdot \\
\cdot & \cdot & \cdot & \cdot & \cdot & \cdot & \cdot \\
0 & 0 & 0 & \cdot & \cdot & x_{n} & 1
\end{array}\right|
$$




$$
\begin{aligned}
& =\left[-x_{1}\left|\begin{array}{cccccc}
1 & x_{2} & 0 & \cdot & \cdot & \cdot \\
x_{3} & 1 & x_{3} & \cdot & \cdot & \cdot \\
\cdot & \cdot & \cdot & \cdot & \cdot & \cdot \\
0 & 0 & 0 & \cdot & x_{n} & 1
\end{array}\right|+(-1)^{n} C_{n+1} x_{1} \cdots x_{n}\right. \\
& - \\
& \div\left|\begin{array}{ccccccc}
1 & x_{1} & 0 & 0 & \cdot & \cdot & \cdot \\
x_{2} & 1 & x_{2} & 0 & \cdot & \cdot & \cdot \\
\cdot & \cdot & \cdot & \cdot & \cdot & \cdot & \cdot \\
0 & 0 & 0 & \cdot & \cdot & x_{n} & 1
\end{array}\right| .
\end{aligned}
$$

The product $x_{1} \cdots x_{n} \rightarrow 0, n \rightarrow \infty$, and the determinant

$$
\left|\begin{array}{ccccccc}
1 & x_{1} & 0 & 0 & 0 & . & \cdot \\
x_{2} & 1 & x_{2} & 0 & . & . & . \\
\cdot & . & . & . & . & . & . \\
0 & 0 & . & . & . & x_{n} & 1
\end{array}\right| \rightarrow\left(x_{1}\right) .
$$

Hence if $C_{n+1}$ is bounded

$$
C_{1}=\frac{-x_{1}\left(x_{2}\right)}{\left(x_{1}\right)}
$$

Similarly if $C_{1}=1$,

It follows that

$$
C_{2}=\frac{-x_{2}\left(x_{3}\right)}{\left(x_{2}\right)}, \quad \text { etc. }
$$

$$
\begin{aligned}
C_{0}: C_{1}: C_{2}: \cdots: C_{n}: \cdots & \\
& =\left(x_{1}\right):-x_{1}\left(x_{2}\right): x_{1} x_{2}\left(x_{3}\right): \cdots:(-1)^{n} x_{1} \cdots x_{n}\left(x_{n+1}\right): \cdots
\end{aligned}
$$

is a system of values of the ratios of $C_{i}$ satisfying the given system of equations. Since $\left(x_{n+1}\right) \rightarrow 1$ as $n \rightarrow \infty$, and $x_{1} \cdots x_{n} \rightarrow 0$ as $n \rightarrow \infty$, it follows that $C_{n} \rightarrow 0$ as $n \rightarrow \infty$. That the above ratios furnish a solution of the equations may be verified by direct substitution, and by making use of the recurrence relation

$$
\left(x_{i}\right)=\left(x_{i+1}\right)-x_{i} x_{i+1}\left(x_{i+2}\right) .
$$

It is then evident that the restriction $C_{i} \neq 0$ may be removed.

The system of ratios for $C_{i}$ may be extended to negative values of $i$ by letting $C_{-n}$ correspond to 


$$
\frac{(-1)^{n}\left(x_{-n+1}\right)}{x_{-n+1} \cdots x_{-1} x_{0}}
$$

This may be verified as before by substitution in the equations. The coeffcients $C_{ \pm n}$ are meromorphic functions of $\lambda$.

In order to obtain a solution of the differential equation, $C_{-n}$ must approach zero. This condition is not fulfilled independently, but depends upon the value of $\mu$, the characteristic exponent. It is evident from the value of $C_{-n}$ that $\left(x_{-n}\right)$ must approach zero, $n \rightarrow \infty$. The product $x_{-n+1} \cdots x_{-1} x_{0}$ which occurs in the denominator tends to zero. Hence the numerator must tend to zero more rapidly to satisfy the requirement. Then $\mu$ must be a root of the equation

$$
\left|\begin{array}{ccccccccc}
\cdot & \cdot & \cdot & \cdot & \cdot & \cdot & \cdot & \cdot & \cdot \\
\cdot & x_{0} & 1 & x_{0} & \cdot & \cdot & \cdot & \cdot & \cdot \\
\cdot & \cdot & x_{1} & 1 & x_{1} & \cdot & \cdot & \cdot & \cdot \\
\cdot & \cdot & \cdot & x_{2} & 1 & x_{2} & \cdot & \cdot & \cdot \\
\cdot & \cdot & . & \cdot & \cdot & \cdot & . & . & .
\end{array}\right|=0
$$

where the determinant is infinite in both directions.

5. The system of equations for $C_{n}$ may be transformed with advantage. Put $C_{n}=f(n)$, and the equation to be transformed is

If $n+\mu=z$,

$$
f(n+1)+f(n-1)=\frac{(n+\mu)^{2}-r^{2}}{\lambda^{2}} f(n) .
$$

$$
f(z-\mu+1)+f(z-\mu-1)=\frac{z^{2}-r^{2}}{\lambda^{2}} f(z-\mu),
$$

or

$$
\phi(z+1)+\phi(z-1)=\frac{z^{2}-r^{2}}{\lambda^{2}} \phi(z),
$$

where $f(z-\mu)=\phi(z)$. If

$$
\phi(z)=\frac{\lambda^{2 z}}{\Pi(z+r) \Pi(z-r)} v_{z}, \quad \Pi(z)=\Gamma(z+1),
$$

there results the equation

$$
\begin{aligned}
& \frac{\lambda^{2 z+2}}{\Pi(z+1+r) \Pi(z+1-r)} v_{z+1}+\frac{\lambda^{2 z-2}}{\Pi(z+r-1) \Pi(z-r-1)} v_{z-1} \\
& =\frac{z^{2}-r^{2}}{\lambda^{2}} \frac{\lambda^{2 z}}{\Pi(z+r) \Pi(z-r)} v_{z},
\end{aligned}
$$


or

$$
\frac{\lambda^{4}}{(z+r)(z+r+1)(z-r)(z-r+1)} v_{z+1}+v_{z-1}=v_{s} .
$$

Changing $z$ into $z+1$ this equation becomes

$$
v_{z}-v_{z+1}=-\frac{\lambda^{4}}{(z+r+1)(z+r+2)(z-r+1)(z-r+2)} v_{z+2} .
$$

If a proper solution of this difference equation is chosen, then

$$
C_{n}=f(n)=\phi(n+\mu)=\frac{\lambda^{2 n+2 \mu}}{\Pi(n+\mu+r) \Pi(n+\mu-r)} v_{n+\mu} .
$$

The difference equation may be written

$$
v_{n+\mu}-v_{n+\mu+1}=-\frac{\lambda^{4}}{(n+\mu+r+1)(n+\mu+r+2)(n+\mu-r+1)(n+\mu-r+2)} v_{n+\mu+2},
$$

or if $u_{n}=v_{n+\mu}=v_{x}$,

$$
u_{n}-u_{n+1}=-\frac{\lambda^{4}}{(n+\mu+r+1)(n+\mu+r+2)(n+\mu-r+1)(n+\mu-r+2)} u_{n+2 .} .
$$

Let $n=0,1,2, \cdots$, and put $y_{n+1}$ for the coefficient of $u_{n+2}$; then there results the system of equations

$$
\begin{aligned}
-u_{0}+u_{1}+y_{1} u_{2} & =0 \\
- & u_{1}+u_{2}+y_{2} u_{3}=0 \\
- & u_{2}+u_{3}+y_{3} u_{4}=0
\end{aligned}
$$

The determinant

$$
\left|\begin{array}{rrrrrrr}
1 & y_{1} & 0 & 0 & . & . & . \\
-1 & 1 & y_{2} & 0 & . & . & . \\
0 & -1 & 1 & y_{3} & 0 & . & . \\
. & . & . & . & . & . & .
\end{array}\right|
$$

is convergent. This may be proved as follows. Let $E_{n}$ denote the determinant of order $n$ formed with the first $n$ rows and columns of this infinite determinant. Then

$$
E_{n}=1+\sum y_{i}+\sum y_{i} y_{i}+\sum y_{i} y_{i} y_{k}+\cdots
$$

where the summations are extended to all products in which no consecutive $y$ 's occur, and the series is continued as far as such products can be formed. 
This expansion may be proved by induction. By expanding $E_{n+2}$ in terms of the last row, it follows that $E_{n+2}=E_{n+1}+y_{n+1} E_{n}$. Moreover $E_{1}=1$, $E_{2}=1+y_{1}, E_{3}=1+y_{1}+y_{2}, E_{4}=1+y_{1}+y_{2}+y_{3}+y_{1} y_{3}$. If the terms of $E_{n}$ are divided into the classes of those which contain $y_{n-1}$ and those which do not, the rule of formation of the terms of $E_{n}$ is verified. To prove that $E_{n}$ approaches a limit as $n \rightarrow \infty$, consider the series

$$
E=1+\sum y_{i}+\sum y_{i} y_{j}+\cdots,
$$

where $i, j, \cdots=1, \cdots, \infty, i \neq j$, etc., and no consecutive $y$ 's occur in any product. This series is absolutely convergent, for if the absolute values $\left|y_{i}\right|$ are taken and the restriction as to non-consecutive $y$ 's removed, the series becomes the expansion of

$$
\left(1+\left|y_{1}\right|\right)\left(1+\left|y_{2}\right|\right) \cdots
$$

which is a convergent product. This is evident since

$$
\sum\left|y_{n}\right|=\left|\lambda^{4}\right| \sum \frac{1}{|(n+\mu+r)(n+\mu+r+1)(n+\mu-r)(n+\mu-r+1)|}
$$

is a convergent series. Hence since $\left|E-E_{n}\right| \rightarrow 0, n \rightarrow \infty, E_{n} \rightarrow E$ which was to be proved.

6. It is found that a solution of the system of equations for $u_{n}$ exists such that $u_{n} \rightarrow 1$ as $n \rightarrow \infty$. If

$$
\left[y_{1}\right]=\left|\begin{array}{rrrrrrr}
1 & y_{1} & 0 & 0 & . & . & . \\
-1 & 1 & y_{2} & 0 & . & . & . \\
0 & -1 & 1 & y_{3} & . & . & . \\
. & . & . & . & . & . & .
\end{array}\right|
$$

this solution will be shown to be $u_{n}=\left[y_{n+1}\right]$. Since the determinant $\left[y_{n+1}\right]=1+\sum y_{i}+\cdots$ where $i>n$, and since the element $y_{p} \rightarrow 0$ as $p \rightarrow \infty$, then $\left[y_{n+1}\right] \rightarrow 1$ as $n \rightarrow \infty$. By expanding $\left[y_{n+1}\right]$ in terms of the first column,

$$
-\left[y_{n+1}\right]+\left[y_{n+2}\right]+y_{n+1}\left[y_{n+3}\right]=0 \text {. }
$$

But this equality is the result of substituting $u_{n}=\left[y_{n+1}\right]$ in the equations for $u_{n}$. Hence $u_{n}=\left[y_{n+1}\right]$ is a solution of the equations, and $u_{n} \rightarrow 1, n \rightarrow \infty$. The ratios of the coefficients $C_{n}$ are then given for all values of $n$ by the expression

$$
C_{n}=\rho \frac{\lambda^{2 n+2 \mu}}{\Pi(n+\mu+r) \Pi(n+\mu-r)}\left[y_{n+1}\right]
$$


where $\rho$ is independent of $n$. These expressions for $C_{n}$ are not equal to those given above ( $(4)$ if $\rho=1$.

It is then evident that $\mu$ must be chosen so that $\left[y_{n}\right] \rightarrow 0$ as $n \rightarrow-\infty$, or $\mu$ must be a solution of the equation

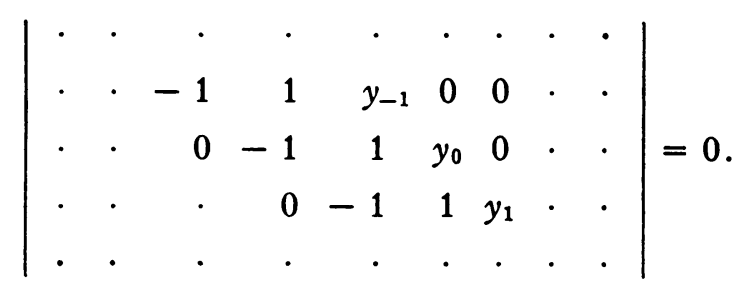

It is necessary to prove the convergence of the series assumed as the solution of the differential equation. For convergence $\mu$ must satisfy the equation already obtained in two forms. It remains to show that this condition is sufficient for convergence. The proof of this fact is postponed until $\$ 11$.

If $(n+\mu)^{2}-r^{2}=0$ the form given for the ratio of the coefficients becomes indeterminate but this indetermination may be removed. For if $p+\mu \pm r=0$, $p>0$, the functions

$$
\frac{(-1)^{n}\left(x_{-n+1}\right)}{x_{-n+1} \cdots x_{-1} x_{0}}, \quad(-1)^{n} x_{1} \cdots x_{n}\left(x_{n+1}\right)
$$

may be multiplied throughout by $p+\mu \pm r$ which gives a set of finite values when $p+\mu \pm r=0$. If $p+\mu \pm r=0, p \leqq 0$, the numerator and denominator of

$$
\frac{(-1)^{n}\left(x_{-n+1}\right)}{x_{-n+1} \cdots x_{-1} x_{0}}
$$

may be multiplied by $p+\mu \pm r$ which gives finite values when $p+\mu \pm r=0$. The equations for $\mu$ will be replaced in the next part of this paper by an equation the terms of which are determinate if $p+\mu \pm r=0$.

\section{THE CHARACTERISTIC EXPONENT}

7. The exponent $\mu$ is the root of a transcendental equation which has been given in what precedes in two different forms. The determination of $\mu$ can however be effected in a different manner, which allows the construction of an explicit expression of at least a branch of $\mu$. It will be proved that $\mu$ is an analytic function with multiple determination of the principal parameter $k^{2}$ of the differential equation. It is found simpler to take the differential equation in the form 


$$
\frac{d^{2} y}{d z^{2}}=\left(\lambda \cos ^{2} z+h^{2}\right) y, \quad \lambda=-k^{2}, \quad h^{2}=-a .
$$

Regarding $h$ as a constant and $\lambda$ as a variable, a branch of $\mu$ will be found as a convergent series of powers of $\lambda$, provided $h$ is not equal to certain exceptiunal values which will be deterr..ined. When $h$ has these exceptional values the proper expression for $\mu$ will likewise be found.

Whatever $h$, the solution of the differential equation will first be proved to be an integral function of $z$ and $\lambda$, expressed as an absolutely convergent series of powers in each. This follows from the determination of the coefficients of a series of powers in $z$ satisfying the differential equation. For if $y=a_{0}+a_{1} z+\cdots$ where $a_{3}$ and $a_{1}$ are arbitrary, the remaining coefficients $a_{2}, \cdots$ are determined by comparison from

$$
2 a_{2}+6 a_{3} z+\cdots=\left(\lambda \cos ^{2} z+h^{2}\right)\left(a_{0}+a_{1} z+a_{2} z^{2}+\cdots\right) .
$$

Hence

$$
\begin{aligned}
2 a_{2} & =h^{2} a_{0} \\
6 a_{3} & =h^{2} a_{1} \\
12 a_{4} & =h^{2} a_{2}+\lambda a_{0}
\end{aligned}
$$

The series for $y$ determined in this way is known to be an integral function of $z$ for all values of $\lambda, h^{2}, a_{0}, a_{1}$. The coefficients $a_{n}$ are polynomials in $\lambda$ whose coefficients depend upon $a_{0}, a_{1}, h^{2}$, and upon the coefficients of the expansion of $\cos ^{2} z$. If a comparison is made with the differential equation

$$
\frac{d^{2} \bar{y}}{d z^{2}}=\left[\lambda(\cos i z)^{2}+\left|h^{2}\right|\right] \bar{y}
$$

and if initial values $\left|a_{0}\right|,\left|a_{1}\right|$ are taken, a series will be found for $\bar{y}$ whose coefficients are polynomials in $\lambda$, which are determined in a manner similar to those above. These polynomials in $\lambda$ have positive coefficients whose values are greater respectively than the absolute values of the corresponding coefficients in the determination of $y$. But the series for $\bar{y}$ is likewise an integral function of $z$. Hence the series for $y$ is one whose terms admit arbitrary redistribution whatever $z, \lambda, a_{0}, a_{1}, h^{2}$, and so is an integral function of $z$ and $\lambda$.

Let $y_{1}=f_{1}(z), y_{2}=f_{2}(z)$ be two independent solutions of the differential equation. Then if

$$
\begin{array}{ll}
y_{1}^{\prime}=f_{1}(z+\pi), & y_{2}^{\prime}=f_{2}(z+\pi), \\
y_{1}^{\prime}=a_{11} y_{1}+a_{12} y_{2}, & y_{2}^{\prime}=a_{21} y_{1}+a_{22} y_{2} .
\end{array}
$$


A solution* $Y_{1}=c_{1} y_{1}+c_{2} y_{2}$ may be found such that

$$
Y_{1}^{\prime}=\nu Y_{1} \quad \text { where } \quad Y_{1}^{\prime}=c_{1} f_{1}(z+\pi)+c_{2} f_{2}(z+\pi) .
$$

The quantity $\nu$ is a root of the equation

$$
\left|\begin{array}{ll}
a_{11}-\nu & a_{21} \\
a_{12} & a_{22}-\nu
\end{array}\right|=\nu^{2}-\left(a_{11}+a_{22}\right) \nu+a_{11} a_{22}-a_{12} a_{21}=0 .
$$

But since the general solution of the differential equation is of the form

$$
y_{1}=c_{1} e^{\mu z} \phi\left(e^{2 z i}\right)+c_{2} e^{-\mu z} \psi\left(e^{2 z i}\right),
$$

the values of $\nu$ must be $e^{\mu \pi}$ and $e^{-\mu \pi}$, so that their product is unity. Hence $a_{11} a_{22}-a_{12} a_{21}=1$. Therefore $\nu$ is a root of the equation

$\nu^{2}-\left(a_{11}+a_{22}\right) \nu+1=0, \quad$ or $\quad 2 \nu=a_{11}+a_{22} \pm\left[\left(a_{11}+a_{22}\right)^{2}-4\right]^{1 / 2}$.

The solution $Y_{1}=c_{1} y_{1}+c_{2} y_{2}$ which satisfies the condition $Y_{1}^{\prime}=\nu Y_{1}$ is then given by $Y_{1}=a_{21} y_{1}+\left(\nu-a_{11}\right) y_{2}$. The solution $Y_{2}=a_{21} y_{1}+\left(1 / \nu-a_{11}\right) y_{2}$ also satisfies the similar condition $Y_{2}^{\prime}=(1 / \nu) Y_{2}$. If $a_{21}=0$, then

$$
Y_{1}=y_{2}, \quad Y_{2}=\left(a_{11}-a_{22}\right) y_{1}+a_{12} y_{2}
$$

are solutions satisfying $Y_{1}^{\prime}=a_{22} y_{1}, Y_{2}^{\prime}=a_{11} y_{2}$.

The coefficients $a_{11}, a_{12}, a_{21}, a_{22}$ are functions of $\lambda$ determined by the two equations

If

$$
\begin{aligned}
& f_{1}(z+\pi)=a_{11} f_{1}(z)+a_{12} f_{2}(z) \\
& f_{2}(z+\pi)=a_{21} f_{1}(z)+a_{22} f_{2}(z) .
\end{aligned}
$$

then

$$
\begin{aligned}
& f_{1}(z) \quad=a_{0}+a_{1} z+\cdots \\
& f_{2}(z) \quad=b_{0}+b_{1} z+\cdots \\
& f_{1}(z+\pi)=A_{0}+A_{1} z+\cdots \\
& f_{2}(z+\pi)=B_{0}+B_{1} z+\cdots
\end{aligned}
$$

$$
\begin{aligned}
& A_{0}=a_{11} a_{0}+a_{12} b_{0}, \\
& B_{0}=a_{21} a_{0}+a_{22} b_{0}, \\
& A_{1}=a_{11} a_{1}+a_{12} b_{1}, \\
& B_{1}=a_{21} a_{1}+a_{22} b_{1},
\end{aligned}
$$

* Goursat, Analyse Mathematique, 2d edition, vol. 2, p. 470. 
and

$$
\begin{array}{ll}
a_{11}=\frac{A_{0} b_{1}-A_{1} b_{0}}{a_{0} b_{1}-a_{1} b_{0}}, & a_{12}=\frac{-A_{0} a_{1}+A_{1} a_{0}}{a_{0} b_{1}-a_{1} b_{0}}, \\
a_{21}=\frac{B_{0} b_{1}-B_{1} b_{0}}{a_{0} b_{1}-a_{1} b_{0}}, & a_{22}=\frac{-B_{0} a_{1}+B_{1} a_{0}}{a_{0} b_{1}-a_{1} b_{0}} .
\end{array}
$$

$a_{0} b_{1}-a_{1} b_{0} \neq 0$, since the solutions $f_{1}$ and $f_{2}$ are independent. Hence $a_{11}$, $a_{12}, a_{21}, a_{22}$ are integral functions of $\lambda$. Since $2 \nu=a_{11}+a_{22} \pm\left[\left(a_{11}+a_{22}\right)^{2}-4\right]^{1 / 2}$, the value of $\nu$ may be written

$$
2 \nu=P(\lambda) \pm\left[\left(a_{11}^{0}+a_{22}^{0}\right)^{2}-4+C_{1} \lambda+C_{2} \lambda^{2}+\cdots\right]^{1 / 2}
$$

where $a_{11}^{0}, a_{22}^{0}$ are the values of $a_{11}, a_{22}$ when $\lambda=0$. If $\left(a^{0}{ }_{1}+a_{22}^{0}\right)^{2}-4 \neq 0$ the radical may be expanded in powers of $\lambda$, and $2 \nu=P(\lambda) \pm P_{1}(\lambda)$. The series $P_{1}(\lambda)$ is convergent if

$$
\left|\frac{C_{1} \lambda+C_{2} \lambda^{2}+\cdots}{\left(a_{11}^{0}+a\right)_{22}^{0}-4}\right|<1,
$$

and a quantity $\sigma$ may be determined such that this condition is fulfilled if $|\lambda|<\sigma$. If $\lambda=0$ the solution of the differential equation is $y=c_{1} e^{h s}+c_{2} e^{-h s}$, and the values of $\nu$ are $e^{h \pi}, e^{-h \pi}$. Hence

$$
a_{11}^{0}+a_{22}^{0}=e^{h \pi}+e^{-h \pi}=2 \cosh h \pi .
$$

If $\left(a_{11}^{0}+a_{22}^{0}\right)^{2}-4=0$, then $e^{h \pi}= \pm 1$, or $h \pi=2 n \pi i$, or $(2 n+1) \pi i$. These give exceptional values of $h$, which will for the present be excluded from the discussion.

The solutions

$$
\begin{aligned}
& Y_{1}=a_{21} y_{1}+\left(\nu-a_{11}\right) y_{2}, \\
& Y_{2}=a_{21} y_{1}+\left(1 / \nu-a_{11}\right) y_{2},
\end{aligned}
$$$$
a_{21} \neq 0 \text {, }
$$

must reduce if $\lambda=0$ to multiples of $e^{h \varepsilon}$ and $e^{-h z}$. The solutions $Y_{1}$ and $Y_{2}$ do not both vanish identically if $\lambda=0$, for since $y_{1}, y_{2}$ do not both vanish identically this would require that $a_{21}=0, \nu-a_{11}=0,1 / \nu-a_{11}=0$. But the last two are contradictory, since $\nu \neq 1 / \nu$ unless $h$ is an exceptional value. Hence

$$
\begin{aligned}
& Y_{1}=m_{1} e^{h z}+\lambda P_{1}(z, \lambda), \\
& Y_{2}=m_{2} e^{-h z}+\lambda P_{2}(z, \lambda),
\end{aligned}
$$

where $m_{1}$ and $m_{2}$ are independent of $z$ and $\lambda$, and $m_{1}$ and $m_{2}$ are not both equal to zero. If $m_{1} \neq 0$, divide by $m_{1}$ and there results a solution of the differential equation 


$$
y=e^{h z}+\lambda \frac{P_{1}(z, \lambda)}{m_{1}}=e^{h z}+\lambda Q_{1}(z, \lambda) .
$$

Let $Z=y^{\prime} / y$ where $y^{\prime}$ denotes differentiation. Then

$$
Z=\frac{h e^{h z}+\lambda Q_{1}^{\prime}(z, \lambda)}{e^{h z}+\lambda Q_{1}(z, \lambda)}=\frac{e^{-h z}\left[h e^{h z}+\lambda Q_{1}^{\prime}(z, \lambda)\right]}{1+\lambda e^{-h z} Q_{1}(z, \lambda)} .
$$

Let $\rho$ be an arbitrary quantity; then $\tau<\sigma$ can now be determined such that

$$
\left|\lambda e^{-h z} Q_{1}(z, \lambda)\right|<1 \text { when }|z|<\rho, \quad|\lambda|<\tau .
$$

Hence if $|z|<\rho,|\lambda|<\tau$, the fraction for $Z$ may be expanded in powers of $\lambda$, or $Z=h+z_{1} \lambda+z_{2} \lambda^{2}+\cdots$. To verify this statement it will be noted that since the series for $a_{21}, \nu, a_{11}, y_{1}, y_{2}$ are all absolutely convergent, if $|\lambda|<\sigma$ and $|z|<\rho$, the expressions for $Y_{1}, Y_{2}$ are absolutely convergent series for the same values of $\lambda$ and $z$. That is, if $Y_{1}=\sum \sum a_{m n} z^{m} \lambda^{n}, \sum \sum\left|a_{m n}\right| z^{m} \lambda^{n}$ is a convergent series, so that an arbitrary arrangement of terms is admissible. Under the same conditions the series for $1+\lambda e^{-h z} Q_{1}(z, \lambda)$ is absolutely convergent because

$$
1+\lambda e^{-h z} Q_{1}(z, \lambda)=e^{-h z}\left[e^{h z}+\lambda Q_{1}(z, \lambda)\right],
$$

and both factors of this product are absolutely convergent. Finally the series for $\left[1+\lambda e^{-h z} Q_{1}(z, \lambda)\right]^{-1}$ is absolutely convergent if $|\lambda|<\tau$ where $\tau$ is determined as follows. In $Q_{1}(z, \lambda)$ let all of the coefficients be replaced by their absolute values and let the resulting series be denoted by $\bar{Q}_{1}(z, \lambda)$. Then determine $\tau<\sigma$ so that $\tau \cdot e^{|h|} \rho \bar{Q}_{1}(\rho, \tau)<1$. Then evidently

$$
\left|\lambda e^{-h z} Q_{1}(z, \lambda)\right|<1 \text { if }|\lambda|<\tau, \quad|z|<\rho .
$$

The series

$$
1+\lambda e^{|h| z} \bar{Q}_{1}(z, \lambda)+\lambda^{2} e^{2|h| z} \bar{Q}_{1}^{2}(z, \lambda)+\cdots
$$

is convergent, and the terms in the expansion of $\lambda^{n} e^{n|h|^{2}} Q_{1^{n}}(z, \lambda)$ have positive coefficients. Hence finally the series

$$
1-h e^{-h z} Q_{1}(z, \lambda)+\lambda^{2} e^{-2 h z} Q_{1}(z, \lambda)-\cdots
$$

is convergent and admits arbitrary rearrangement of terms.

8. If $Z=y^{\prime} / y, Z$ satisfies the Riccati equation $Z^{\prime}+Z^{2}=\lambda \cos ^{2} z+h^{2}$, and a solution $Z$ of this equation has been found. If this solution is $Z=h+Z_{1} \lambda$ $+Z_{2} \lambda^{2}+\cdots$, the coefficients $Z_{1}, Z_{2}$, etc. may now be determined by substitution in the differential equation, and by comparison of coefficients of like powers of $\lambda$. There results the system of equations 


$$
\begin{aligned}
& Z_{1}^{\prime}+2 h Z_{1}=\cos ^{2} z \\
& Z_{2}^{\prime}+2 h Z_{2}+Z_{1}^{2}=0 \\
& Z_{3}^{\prime}+2 h Z_{3}+2 Z_{1} Z_{2}=0 \\
& Z_{4}^{\prime}+2 h Z_{4}+Z_{2}^{2}+2 Z_{1} Z_{3}=0 \\
& Z_{5}^{\prime}+2 h Z_{5}+2 Z_{1} Z_{4}+2 Z_{1} Z_{3}=0
\end{aligned}
$$

This is a system of equations which may be solved, the complementary function in each case being $c e^{-2 h z}$. But the solution $Z$ is a periodic function of $z$ with period $\pi$. Then the coefficients $Z_{1}$ and $Z_{2}$ must be periodic, and since the particular solutions of the equations for $Z$ are periodic, the complementary functions must in every case be omitted. It is found that

$$
Z_{1}=\frac{1}{2^{2} h}+\frac{\sin 2 z+h \cos 2 z}{2^{2}\left(1+h^{2}\right)},
$$

and in general $Z_{n}$ equals a finite series of sines and cosines of even multiples up to $2 n z$, i.e.

$Z_{n}=b_{n, 1}+b_{n, 2} \sin 2 z+b_{n, 8} \cos 2 z+\cdots+b_{n, 2 n} \sin 2 n z+b_{n, 2 n+1} \cos 2 n z$,

where the coefficients $b_{i j}$ are rational functions of $h$.

9. The general solution of the differential equation

$$
\frac{d^{2} y}{d z^{2}}=\left(\lambda \cos ^{2} z+h^{2}\right) y
$$

may be taken in the form

$$
y=c_{1} e^{2 \mu i z} \phi\left(e^{2 z i}\right)+c_{2} e^{-2 \mu i z} \psi\left(e^{2 z i}\right),
$$

where $\phi$ and $\psi$ are series of positive and negative powers of $e^{2 z i}$. It is evident that $\nu=e^{2 \mu \pi i}$, or $e^{-2 \mu \pi i}$. The Riccati equation $Z^{\prime}+Z^{2}=\lambda \cos ^{2} z+h^{2}$ is satisfied by $y^{\prime} / y$ whatever $c_{1}$ and $c_{2}$. If $c_{1}=0$, or $c_{2}=0, y^{\prime} / y$ is a periodic function of $z$ of period $\pi$ and in no other case. Hence the function $Z$ of the preceding discussion must correspond to one of these cases, and since the sign of $\mu$ has not been fixed, it may be supposed that it corresponds to $c_{2}=0$. Hence $Z=2 \mu i+\phi^{\prime} / \phi$, where $\phi^{\prime}$ denotes differentiation with respect to $z$. Therefore

$$
2 \mu i+\phi^{\prime} / \phi=h+Z_{1} \lambda+Z_{2} \lambda^{2}+\cdots .
$$

The series on the right has been determined and is a uniformly convergent series of functions of $z$ if $|\lambda|<\tau,|z|<\rho$. Since $\rho$ is arbitrary it may be supposed that $\rho>\pi$. Integration with respect to $z$ from 0 to $\pi$ gives 


$$
2 \pi \mu i+\int_{0}^{\pi} \frac{\phi^{\prime}}{\phi} d z=\pi h+\lambda \int_{0}^{\pi} Z_{1} d z+\cdots
$$

The value of

$$
\int_{0}^{\pi} Z_{n} d z=\pi b_{n, 1}
$$

since the integrals of the trigonometric terms vanish. Hence

But

$$
2 \mu i+\frac{1}{\pi} \int_{0}^{\pi} \frac{\phi^{\prime}}{\phi} d z=h+\sum_{n=1 \ldots \infty} b_{n, 1} \lambda^{n} .
$$

$$
\left.\int_{0}^{\pi} \frac{\phi^{\prime}}{\phi} d z=\frac{1}{\pi} \log \phi\right]_{0}^{\pi}=\frac{\log 1}{\pi}=2 n i,
$$

since $\phi$ has the period $\pi$. Finally

$$
2 \mu i+2 n i=h+\sum_{n=1 \ldots \infty} b_{n, 1} \lambda^{n},
$$

a series for $\mu$ convergent if $|\lambda|<\tau$. The integer $n$ is in the nature of the case not determined, since in the form of the general solution of the differential equation $2 \mu i$ may be increased by $2 n i$.

A similar series for $\mu$ will be found if the differential equation is taken in the hyperbolic form

$$
\frac{d^{2} y}{d z^{2}}=-\left(h^{2}+\lambda \cosh ^{2} z\right) y .
$$

Substitute $z=i z^{\prime}$ and the equation becomes

$$
\frac{d^{2} y}{d z^{\prime 2}}=\left(h^{2}+\lambda \cos ^{2} z^{\prime}\right) y .
$$

This equation shows that a series giving $\mu$ for the hyperbolic form may be derived from the series which gives $\mu$ for the trigonometric form by changing the sign of $\lambda$ and replacing $h^{2}$ by $-h^{2}$. For the trigonometric form of the equation $\mu$ is given by the series

$$
\begin{aligned}
& 2 i(\mu+n)=h+\frac{1}{2^{2} h} \lambda-\frac{3 h^{2}+2}{2^{6} h^{3}\left(1+h^{2}\right)} \lambda^{2}+\frac{5 h^{6}+10 h^{4}+7 h^{2}+2}{28 h^{5}\left(1+h^{2}\right)^{3}} \lambda^{3} \\
& -\frac{175 h^{12}+1750 h^{10}+6027 h^{8}+8876 h^{6}+7048 h^{4}+3264 h^{2}+640}{2^{14} h^{7}\left(1+h^{2}\right)^{4}\left(4+h^{2}\right)^{2}} \lambda^{4} \\
& +
\end{aligned}
$$


For the hyperbolic form of the equation $\mu$ is given by the series

$$
2 i(\mu+n)=i h-\frac{1}{2^{2} i h} \lambda+\frac{-3 h^{2}+2}{2^{6} i h^{3}\left(1-h^{2}\right)} \lambda^{2}+\cdots
$$

It is evident that within the range of convergence of these series the value of $\mu$ is real for the hyperbolic case and a pure imaginary for the trigonometric.

If $h$ has one of the exceptional values $0, \pm n i$ the equations for $Z_{i}$ take a different form. Suppose $h=0$; then $Y_{1}, Y_{2}$ are series of powers of $\lambda^{1 / 2}$ $(\$ 7)$ and

$$
Z=z_{1} \lambda^{1 / 2}+z_{2} \lambda+\cdots \cdot
$$

The differential equation $Z^{\prime}+Z^{2}=\lambda \cos ^{2} z$ gives the system

$$
\begin{aligned}
& Z_{1}^{\prime}=0, \\
& Z_{2}^{\prime}+Z_{1}^{2}=\cos ^{2} z, \\
& Z_{3}^{\prime}+2 Z_{1} Z_{2}=0, \\
& Z_{4}^{\prime}+Z_{2}^{2}+2 Z_{1} Z_{3}=0,
\end{aligned}
$$

These equations must have a periodic solution. The first equation gives $Z_{1}=c$, and substituting in the second it is seen that

$$
Z_{2}=\left(-c^{2}+\frac{1}{2}\right) z+\frac{\sin 2 z}{4}+c^{\prime}
$$

hence $c=1 / 2^{1 / 2}$. Similarly

$$
Z_{3}=\frac{2^{1 / 2} \cos 2 z}{8}-2^{1 / 2} c^{\prime} z+c^{\prime \prime}
$$

and $c^{\prime}=0$. If this value of $Z_{3}$ is substituted in the next equation

$$
Z_{4}=-\left(\frac{1}{32}+2^{1 / 2} c^{\prime \prime}\right) z+\frac{\sin 4 z}{128}-\frac{\sin 2 z}{8}+c^{\prime \prime \prime} .
$$

Hence $c^{\prime \prime}=-1 /\left(2^{1 / 2} \cdot 32\right)$, etc. Finally

$$
\begin{gathered}
Z=\frac{\lambda^{1 / 2}}{2^{1 / 2}}+\frac{\sin 2 z}{4} \lambda+\left(\frac{2^{1 / 2} \cos 2 z}{8}-\frac{1}{2^{1 / 2} \cdot 32}\right) \lambda^{3 / 2} \\
+\left(\frac{\sin 4 z}{128}-\frac{\sin 2 z}{8}\right) \lambda^{2}+\cdots
\end{gathered}
$$


The value of $\mu$ may be found as before and is given by

$$
2 \pi i(\mu+n)=\frac{\lambda^{1 / 2}}{2^{1 / 2}}-\frac{\lambda^{3 / 2}}{2^{1 / 2} \cdot 32}+\cdots .
$$

When $h$ has one of the other exceptional values it becomes necessary to have recourse to the equation given hereafter for $\mu(\S 10)$, the coefficients of which become indeterminate for the exceptional values, but the indetermination can be removed in the usual way.

10. It will now be proved that the exponent $\mu$ may be determined by an equation involving a series convergent for all values of $\lambda$. It has been shown (§7) that $u_{n}=\left[y_{n+1}\right]$ satisfies the equation

$$
u_{n}-u_{n+1}=y_{n+1} u_{n+2} \text {. }
$$

If $u_{n}=v_{n+\mu}=v_{z}$ then

If

$$
v_{z}-v_{z+1}=-\frac{\lambda^{4}}{(z+r+1)(z+r+2)(z-r+1)(z-r+2)} v_{s+2} .
$$

$$
\phi(z)=\frac{\lambda^{2 z}}{\Pi(z+r) \Pi(z-r)} v_{z},
$$

then $\phi(z)$ satisfies the difference equation

If

$$
\phi(z+1)+\phi(z-1)=\frac{z^{2}-r^{2}}{\lambda^{2}} \phi(z) .
$$

$$
f(z)=\phi(z) \phi(-z-1)-\phi(-z) \phi(z+1),
$$

then it may be shown that $f(z)=f(z-1)=f(-z)$. This is evident since $\phi(z)$ satisfies the equation above. If the sign of $z$ is changed, $\phi(z)$ satisfies the equation

$$
\phi(-z+1)+\phi(-z-1)=\frac{z^{2}-r^{2}}{\lambda^{2}} \phi(-z) .
$$

If $\left(z^{2}-r^{2}\right) / \lambda^{2}$ is eliminated the eliminant is

$$
\phi(z) \phi(-z-1)-\phi(-z) \phi(z+1)=\phi(z-1) \phi(-z)-\phi(-z+1) \phi(z) \text {, }
$$

or $f(z)=f(z-1)$. It is evident at once that $f(z)=f(-z)$. If $z$ is replaced in turn by $\mu$ and by $n+\mu$ it follows that

$$
\begin{aligned}
\phi(\mu) \phi(-\mu-1) & -\phi(-\mu) \phi(\mu+1)=\phi(n+\mu) \phi(-n-\mu-1) \\
& -\phi(-n-\mu) \phi(n+\mu+1) .
\end{aligned}
$$


If $\mu$ is a solution of the equations $(\$ \S 4,6)$

and

$$
\left|\begin{array}{ccccccc}
\cdot & \cdot & . & . & . & . & . \\
\cdot & . & x_{0} & 1 & x_{0} & . & . \\
\cdot & . & . & x_{1} & 1 & x_{1} & . \\
. & . & . & . & . & . & .
\end{array}\right|=0
$$

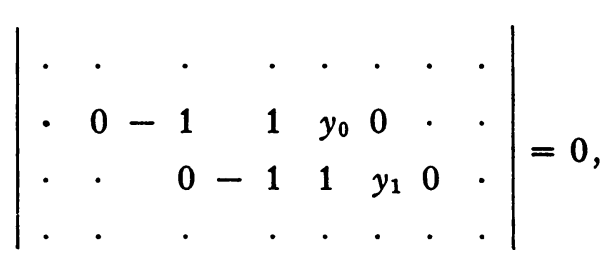

the quantities $\phi(n+\mu), \phi(-n-\mu) \rightarrow 0$ as $n \rightarrow \infty$. This is evident for $\phi(n+\mu)$. It is also true for $\phi(-n-\mu)$, because from the form of the equations for $\mu$ it is seen that if $\mu$ is a root of the equations, $-\mu$ is also a root. $\mu$ is in fact a value such that $\phi(n-\mu) \rightarrow 0, \phi(-n-\mu) \rightarrow 0$ when $n \rightarrow \infty$. The function $f(\mu)$ must then equal zero, or $\mu$ satisfies the equation

$$
\phi(\mu) \phi(-\mu-1)-\phi(-\mu) \phi(\mu+1)=0 .
$$

This equation in $\mu$ if transformed by the introduction of the function $v_{z}$ takes a simple form. For since

$$
\begin{gathered}
(z)=\lim _{n \rightarrow-\infty}\left\{\frac{\lambda^{2 z}}{\phi(z)=\frac{\lambda^{-2} v_{n+z_{-n-z-1}}}{\pi(z+r) \pi(z-r)} v_{z},}\right. \\
\left.-\frac{\lambda^{2} v_{-n-z} v_{n+z+1}}{\Pi(-n-z+r) \Pi(-n-z-r) \Pi(n+z+1+r) \Pi(n+z+1-r)}\right\} .
\end{gathered}
$$

Now if $n \rightarrow-\infty, v_{-n-2}$ and $v_{-n-z-1} \rightarrow 1$, and

$$
v_{n+z} \rightarrow\left|\begin{array}{ccccccccc}
. & . & . & . & . & . & . & . & . \\
. & . & -1 & 1 & y_{-1} & 0 & . & . & . \\
. & . & 0 & -1 & 1 & y_{0} & . & . & . \\
. & . & 0 & 0 & -1 & 1 & y_{1} & . & . \\
. & . & . & . & . & . & . & . & .
\end{array}\right| \text {, }
$$

the infinite determinant of $\S 6$. Moreover, since

$$
\Pi(z) \Pi(-z-1)=\frac{-\pi}{\sin \pi z},
$$


then

$$
\begin{gathered}
\frac{\lambda^{-2}}{\Pi(n+z+r) \Pi(n+z-r) \Pi(-n-z-1+r) \Pi(-n-z-1-r)} \\
=\frac{\sin \pi(z+r) \sin \pi(z-r)}{\pi^{2} \lambda^{2}},
\end{gathered}
$$

and

$$
\begin{aligned}
\frac{\lambda^{2}}{\Pi(-n-z+r) \Pi(-n-z-r) \Pi(n+z+1+r) \Pi(n+z-1-r)} \\
=\frac{\lambda^{2}}{\pi^{2}(n+z+1+r)(n+z+r)(n+z+1-r)(n+z-r)} .
\end{aligned}
$$

If these results are substituted in the expression for $f(z)$ it follows that

$$
f(z)=\frac{\sin \pi(z+r) \sin \pi(z-r)}{\pi^{2} \lambda^{2}}\left|\begin{array}{rrrrrrrr}
\cdot & \cdot & \cdot & \cdot & . & . & \cdot & \cdot \\
\cdot & \cdot & -1 & 1 & y_{-1} & 0 & \cdot & \cdot \\
\cdot & \cdot & 0 & -1 & 1 & y_{0} & \cdot & \cdot \\
\cdot & \cdot & 0 & 0 & -1 & 1 & y_{1} & \cdot \\
\cdot & \cdot & . & . & . & . & . & .
\end{array}\right|=0 .
$$

The determinant may be developed in a series of powers of $\lambda$, the coefficients of which are of the form given by the construction of $\left[y_{n}\right]$. The equation for $\mu$ takes the form

$$
\left(1+a_{4} \lambda^{4}+a_{8} \lambda^{8}+\cdots\right) \sin \pi(\mu+r) \sin \pi(\mu-r)=0 .
$$

This may be written $\cos 2 \pi \mu=\cos 2 \pi r-2 b_{4} \lambda^{4}+2 b_{8} \lambda^{8}-\cdots$, where $b_{4}=$ $-a_{4} \sin \pi(\mu+r) \sin \pi(\mu-r)$, and similarly for $b_{8}, \cdots$.

The coefficients $b_{4}, \cdots$ are independent of $\mu$. The coefficients $a_{4 q}$ may be written

$$
(-1)^{q} \sum_{p_{1}=-\infty}^{+\infty} \cdots \sum_{p_{q}=2}^{\infty} y_{p_{1}} x_{p_{1}+p_{2}} \cdots y_{p_{1}+p_{2}}+\cdots+p_{q} .
$$

The function of $\mu$ under the summation sign is a rational function of $\mu$ having $4 q$ poles for $2 q$ of which $\mu+r$ is an integer, and for the other $2 q$, $\mu-\boldsymbol{r}$ is an integer. If a residue at a pole for which $\mu-r$ is an integer is $d$, the residue at a pole for which $\mu+r$ is an integer is $-d$, since $a_{1 q}$ is periodic with period unity. Hence

$$
a_{4 q}=(-1)^{q} \pi[\cot \pi(\mu-r)-\cot \pi(\mu+r)] d+C,
$$

where $C$ is independent of $\mu$. If $\mu=i l$, then as $l \rightarrow \infty, a_{4 q} \rightarrow 0, \cot \pi(\mu-r)$ $-\cot \pi(\mu+r) \rightarrow 0$, so $C=0$. Hence 


$$
a_{4 q}=\frac{(-1)^{q} \sin 2 r \pi}{\sin \pi(\mu+r) \sin \pi(\mu-r)} d,
$$

or $b_{4 q}=(-1)^{q} d \sin 2 r \pi$, which is independent of $\mu$.

The form of the coefficients $b$ may be found more precisely as follows. Let $\mu=r+c_{1} \lambda^{4}+\cdots=r+w$, where the coefficients are those of the branch of $\mu$ previously determined ( $\$ 9$ ); then

$$
\begin{aligned}
\cos 2 \pi(r+w)= & \cos 2 \pi r \cos 2 \pi w-\sin 2 \pi r \sin 2 \pi w \\
= & \cos 2 \pi r\left\{1-\frac{(2 \pi w)^{2}}{2}+\cdots\right\} \\
& \quad-\sin 2 \pi r\left\{2 \pi w-\frac{(2 \pi w)^{3}}{6}+\cdots\right\} .
\end{aligned}
$$

If $w$ is replaced by its value as a series in $\lambda$ the coefficients $b$ may be determined, and they are found to be of the form

$$
b_{4 q}=C_{q}(r) \cos 2 \pi r+S_{q}(r) \sin 2 \pi r
$$

where $C_{q}(r), S_{q}(r)$ are rational functions of $r$. Hence finally

$$
\cos 2 \pi \mu=\cos 2 \pi r+\sum_{q=1}^{\infty}(-1)^{q}\left\{C_{q}(r) \cos 2 \pi r+S_{q}(r) \sin 2 \pi r\right\} \lambda^{4 q} .
$$

This equation is valid for all values of $\lambda$.

If $r$ has one of the exceptional values, viz. if $2 r=0, \pm n$ the form of the functions $C_{q}, S_{q}$ must be modified. The modification is found by evaluating the coefficients $C_{q}(r), S_{Q}(r)$ which are indeterminate. If $r=0$ the corresponding branch of $\mu$ which is found in $\$ 8$ may be substituted in the equation for $\mu$ giving in another way the result for this case.

11. The proof of the convergence of the series $e^{2 \mu z} \sum_{-\infty}^{+\infty} c_{n} e^{2 n z}$ which was postponed in $\S 6$ may now be given. Consider the terms of the series $\sum_{1}^{\infty} c_{n} e^{2 n s}$. The ratio of the $(n+1)$ st term to the $n$th gives

$$
\frac{c_{n+1} e^{2(n+1) z}}{c_{n} e^{2 n z}}=\frac{x_{n+1}\left(x_{n+2}\right) e^{2 \varepsilon}}{\left(x_{n+1}\right)}
$$

(see $\S 4)$. From the expansion of $\left(x_{n}\right)$ in a series

$$
\left(x_{n}\right)=1-\sum_{i} x_{i} x_{i+1}+\sum_{i>i+1} x_{i} x_{i+1} x_{j} x_{i+1}-\cdots
$$

and from the value of $x_{i}$,

$$
x_{i}=\frac{-\lambda^{2}}{(n+\mu)^{2}-r^{2}}
$$


it is evident that $\left(x_{n}\right) \rightarrow 1, n \rightarrow \infty$. Hence

$$
\frac{c_{n+1}}{c_{n}} e^{2 z}=\frac{\lambda^{2}}{(n+1+\mu)^{2}-r^{2}} e^{2 z}(1+\epsilon) \rightarrow 0, n \rightarrow \infty,
$$

for every value of $z$. Therefore the part of the series where $n$ is positive is convergent.

The series $\sum_{0}^{\infty} c_{-n} e^{-2 n z}$ is likewise convergent, $\mu$ being a root of the equation

$$
\begin{gathered}
\phi(\mu) \phi(-\mu-1)-\phi(-\mu) \phi(\mu+1)=\phi(n-\mu) \phi(-n+\mu-1) \\
-\phi(-n+\mu) \phi(n-\mu+1)=0 ;
\end{gathered}
$$

because the ratio

$$
\begin{aligned}
\frac{c_{-n-1}}{c_{-n}} e^{-2 z} & =\frac{\phi(-n+\mu-1)}{\phi(-n+\mu)} e^{-2 z}=\frac{\phi(n-\mu+1)}{\phi(n-\mu)}-e^{-2 z} \\
& =\frac{\lambda^{2}}{(n+1-\mu)^{2}-r^{2}} e^{-2 z}(1+\epsilon) \rightarrow 0, n \rightarrow \infty .
\end{aligned}
$$

Hence the series $e^{2 \mu z} \sum_{-\infty}^{\infty} c_{n} e^{2 n z}$ is convergent for all values of $z$ and therefore satisfies the differential equation.

12. The form of the equation

$$
\cos 2 \pi \mu=\cos 2 \pi r+\sum_{n=1}^{\infty}(-1)^{n}\left\{C_{n}(r) \cos 2 \pi r+S_{n}(r) \sin 2 \pi r\right\} \lambda^{4 n}
$$

permits the determination of the character of the singular points of $\mu$ regarded as a function of $\lambda$. The singular points will evidently be those values of $\lambda$ which make the right hand side equal to \pm 1 . Let $\lambda_{0}$ be such a value. Then the equation becomes

$$
\cos 2 \pi \mu= \pm 1-a_{1}\left(\lambda-\lambda_{0}\right)+\cdots,
$$

or taking the plus sign

$$
2 \sin ^{2} \pi \mu=a_{1}\left(\lambda-\lambda_{0}\right)+\cdots .
$$

Therefore $\sin \pi \mu=\left(a_{1} / 2\right)^{1 / 2}\left(\lambda-\lambda_{0}\right)^{1 / 2}\left[1+b_{1}\left(\lambda-\lambda_{0}\right)+\cdots\right]$. By inversion of series

$$
\mu=c_{1}\left(\lambda-\lambda_{0}\right)^{1 / 2}+c_{3}\left(\lambda-\lambda_{0}\right)^{3 / 2}+\cdots .
$$

Hence the point $\lambda_{0}$ has the character of a critical point. If $\cos 2 \pi \mu=-1-$ $a_{1}\left(\lambda-\lambda_{0}\right)+\cdots$ a similar result follows. 


\section{ThE PERIODIC SOLUTIONS}

13. That the differential equation of the elliptic cylinder has solutions in certain cases which are not of the form which has been supposed as the general one is usually assumed. A proof of the existence of these solutions is given in $\S 17$. These solutions occur when the parameters $\lambda$ and $r$ have particular relations to each other, and there exists a solution which is periodic with period $\pi i$ or $2 \pi i$. Taking the equation in the trigonometric form it will be written in what follows as

$$
\frac{d^{2} y}{d z^{2}}+\left(a+k^{2} \cos ^{2} z\right) y=0 .
$$

The problem is to determine $a$ in terms of $k$ so that the equation has a solution which has the period $\pi$ or $2 \pi$. It was proved by Whittaker that the even periodic functions satisfy the integral equation

$$
y(z)+\lambda \int_{-\pi}^{+\pi} e^{k \cos z \cos \theta} y(\theta) d \theta=0,
$$

that the odd periodic functions satisfy the equation

$$
y(z)+\lambda \int_{-\pi}^{+\pi} \sin (k \sin z \cos \theta) y(\theta) d \theta=0,
$$

and that both satisfy the equation

$$
y(z)+\lambda \int_{-\pi}^{+\pi} e^{i k \sin z \sin \theta} y(\theta) d \theta=0 .
$$

These equations have solutions only when $\lambda$ satisfies the equation $D(\lambda)=0$, the equation for the characteristic numbers in the theory of integral equations.

When $k=0$ the solution of the differential equation of period $\pi$ or $2 \pi$ is obtained by supposing $a$ to be the square of an integer; these solutions being $1, \cos z, \sin z, \cos 2 z, \sin 2 z, \cdots$. When $k \neq 0$ there exist solutions for proper values of $a$ which reduce to these if $k=0$. These are denoted by $\mathrm{ce}_{0}(z), \mathrm{ce}_{1}(z), \mathrm{se}_{1}(z), \mathrm{ce}_{2}(z), \mathrm{se}_{2}(z)$, etc.

These solutions could be calculated from the integral equation if $\lambda$ were known. But it was shown by Whittaker that the early members of the succession of functions can be found without knowing $\lambda$. His method succeeds as far as $c_{6}(z), \mathrm{se}_{6}(z)$ but cannot be carried further, since to obtain the terms of the Fourier series for $\operatorname{ce}_{n}(z), \mathrm{se}_{n}(z), n \geqq 7$, requires the solution of a system of linear equations which involves a greater number of unknowns 
than of equations. This system might be extended to an infinite one but it is not possible to obtain the solution. Another method must be followed which is exemplified by the following determination of $\operatorname{ce}_{0}(z)$.

14. Let

$$
y=\operatorname{ce}_{0}(z)=a_{0}+a_{2} \cos 2 z+a_{4} \cos 4 z+\cdots
$$

be a solution of the differential equation. Then differentiating and substituting there results

$-a\left(a_{0}+a_{2} \cos 2 z+a_{4} \cos 4 z+\cdots\right)=-2^{2} a_{2} \cos 2 z$

$-4^{2} a_{4} \cos 4 z-\cdots+k^{2}\left[a_{0} / 2+\left(a_{0} / 2\right) \cos 2 z+a_{2} / 4+\left(a_{2} / 4\right) \cos 2 z\right.$

$\left.+\cdots+\left(a_{2 r} / 4\right) \cos (2 r-2) z+\left(a_{2 r} / 2\right) \cos 2 r z+\left(a_{2 r} / 4\right) \cos (2 r+2) z+\cdots\right]$.

Equate coefficients of cosines of like multiples of $z$ and the following equations are obtained:

$$
\begin{aligned}
& \left(a+k^{2} / 2\right) a_{0}+\left(k^{2} / 4\right) a_{2}=0, \\
& \left(k^{2} / 2\right) a_{0}+\left(a+k^{2} / 2-2^{2}\right) a_{2}+\left(k^{2} / 4\right) a_{4}=0 \text {, } \\
& \left(k^{2} / 4\right) a_{2}+\left(a+k^{2} / 2-4^{2}\right) a_{4}+\left(k^{2} / 4\right) a_{6}=0 \text {, } \\
& \left(k^{2} / 4\right) a_{4}+\left(a+k^{2} / 2-6^{2}\right) a_{6}+\left(k^{2} / 4\right) a_{8}=0 \text {, } \\
& \left(k^{2} / 4\right) a_{2 n-2}+\left(a+k^{2} / 2-4 n^{2}\right) a_{2 n}+\left(k^{2} / 4\right) a_{2 n+2}=0 \text {, }
\end{aligned}
$$

It is necessary to consider the determinants

$$
\begin{aligned}
D_{1} & =a+k^{2} / 2, \\
D_{2} & =\left|\begin{array}{cc}
a+k^{2} / 2 & k^{2} / 4 \\
k^{2} / 2 & a+k^{2} / 2-2^{2}
\end{array}\right|=\left(a+k^{2} / 2\right)^{2}-2^{2}\left(a+k^{2} / 2\right)-2(k / 2)^{4}, \\
D_{3} & =\left|\begin{array}{ccc}
a+k^{2} / 2 & k^{2} / 4 & 0 \\
k^{2} / 2 & a+k^{2} / 2-2^{2} & k^{2} / 4 \\
0 & k^{2} / 4 & a+k^{2} / 2-4^{2}
\end{array}\right| \\
& =\left(a+k^{2} / 2\right)^{3}-\left(2^{2}+4^{2}\right)\left(a+k^{2} / 2\right)^{2}+\left(2^{2} 4^{2}-3(k / 2)^{4}\right)\left(a+k^{2} / 2\right) \\
D_{4} & =\left|\begin{array}{cccc}
a+k^{2} / 2 & k^{2} / 4 & 0 & 0 \\
k^{2} / 2 & a+k^{2} / 2-2^{2} & k^{2} / 4 & 0 \\
0 & k^{2} / 4 & a+k^{2} / 2-4^{2} & k^{2} / 4 \\
0 & 0 & k^{2} / 4 & a+k^{2} / 2-6^{2}
\end{array}\right|
\end{aligned}
$$




$$
\begin{aligned}
& =\left(a+k^{2} / 2\right)^{4}-\left(2^{2}+4^{2}+6^{2}\right)\left(a+k^{2} / 2\right)^{3} \\
& +\left(2^{2} \cdot 4^{2}+2^{2} \cdot 6^{2}+4^{2} \cdot 6^{2}-4(k / 2)^{4}\right)\left(a+k^{2} / 2\right)^{2} \\
& -\left\{2^{2} \cdot 4^{2} \cdot 6^{2}-\left(2^{2}+2 \cdot 4^{2}+3 \cdot 6^{2}\right)(k / 2)^{4}\right\}\left(a+k^{2} / 2\right) \\
& -2 \cdot 4^{2} \cdot 6^{2}(k / 2)^{4}+2(k / 2)^{8} \text {, } \\
& D_{6}=\left|\begin{array}{ccccc}
a+k^{2} / 2 & k^{2} / 4 & 0 & 0 & 0 \\
k^{2} / 2 & a+k^{2} / 2-2^{2} & k^{2} / 4 & 0 & 0 \\
0 & k^{2} / 4 & a+k^{2} / 2-4^{2} & k^{2} / 4 & 0 \\
0 & 0 & k^{2} / 4 & a+k^{2} / 2-6^{2} & k^{2} / 4 \\
0 & 0 & 0 & k^{2} / 4 & a+k^{2} / 2-8^{2}
\end{array}\right| \\
& =\left(a+k_{2} / 2\right)^{5}-\left(2^{2}+4^{2}+6^{2}+8^{2}\right)\left(a+k^{2} / 2\right)^{4} \\
& +\left\{2^{2} \cdot 4^{2}+2^{2} \cdot 6^{2}+4^{2} \cdot 6^{2}+2^{2} \cdot 8^{2}+4^{2} \cdot 8^{2}+6^{2} \cdot 8^{2}\right. \\
& \left.-5(k / 2)^{4}\right\}\left(a+k^{2} / 2\right)^{3}-\left\{2^{2} \cdot 4^{2} \cdot 6^{2}+2^{2} \cdot 4^{2} \cdot 8^{2}+2^{2} \cdot 6^{2} \cdot 8^{2}\right. \\
& \left.+4^{2} \cdot 6^{2} \cdot 8^{2}-\left(2 \cdot 2^{2}+3 \cdot 4^{2}+3 \cdot 6^{2}+4 \cdot 8^{2}\right)(k / 2)^{4}\right\}\left(a+k^{2} / 2\right)^{2} \\
& +\left\{2^{2} \cdot 4^{2} \cdot 6^{2} \cdot 8^{2}-\left(2^{2} \cdot 4^{2}+2^{2 \cdot} \cdot 8^{2}+2^{2} \cdot 4^{2} \cdot 6^{2}+2^{2} \cdot 4^{2} \cdot 8^{2}\right.\right. \\
& \left.\left.+3^{2} \cdot 6^{2} \cdot 8^{2}\right)(k / 2)^{4}-5(k / 2)^{8}\right\}\left(a+k^{2} / 2\right) \\
& +2 \cdot 4^{2} \cdot 6^{2} \cdot 8^{2}(k / 2)^{4}-\left(2 \cdot 4^{2}+2 \cdot 8^{2}\right)(k / 2)^{8} \text {. }
\end{aligned}
$$

These determinants are connected by the relation

$$
D_{n}=\left\{a+k^{2} / 2-(2 n-2)^{2}\right\} D_{n-1}-\left(k^{2} / 4\right)^{2} D_{n-2},
$$

as may be verified by expanding $D_{n}$ in terms of the last row. The quantities $D_{n}$ regarded as functions of $a$ have the characteristics of a Sturmian series. $D_{n}$ is of the $n$th degree in $a$, and the coefficient of $a^{n}$ is +1 . Moreover it is evident from the recurrence relation if $D_{n-1}=0$ that $D_{n}$ and $D_{n-2}$ have opposite signs. Denote the number of variations of sign when $a$ is put $=+\infty$ in the succession $D_{n} D_{n-1} \cdots D_{1} D_{0}\left(D_{0}=1\right)$ by $V_{+\infty}$ and when $a=-\infty$ by $V_{-\infty}$, and it is evident that $V_{-\infty}-V_{+\infty}=n$. Hence the equation $D_{n}=0$ has $n$ real roots; it also follows that $D_{n}$ and $D_{n-1}$ have unlike signs just before each of the $n$ roots of $D_{n}=0$ and like signs immediately after.

It will be proved in $\S 17$ that the quantities $a, a_{0}, a_{2}, \cdots$ are power series in $k^{2}$ where $a_{0}$ may be put equal to unity. If it be assumed that a solution $\operatorname{ce}_{0}(z)$ exists which reduces to unity when $k=0$, it follows that $a=\left(k^{2}\right)$, $a_{2}=\left(k^{2}\right), \cdots, a_{2 n}=\left(k^{2}\right)$, where the notation $\left(k^{n}\right)$ means divisible by $k^{n}$. Since $a+k^{2} / 2=-\left(k^{2} / 4\right) a_{2}$, then $a+k^{2} / 2=\left(k^{4}\right)$. If now the solution of the first $n$ equations is expressed in the form 


$$
\begin{aligned}
& a_{0}: a_{2}: \cdots: a_{2 n} \\
& \left\|\begin{array}{ccccccc}
a+k^{2} / 2 & k^{2} / 4 & 0 & \cdot & \cdot & \cdot & \cdot \\
k^{2} / 2 & a+k^{2} / 2-2^{2} & k^{2} / 4 & \cdot & \cdot & \cdot & \cdot \\
0 & k^{2} / 4 & a+k^{2} / 2-4^{2} & \cdot & \cdot & \cdot \\
\cdot & \cdot & \cdot & \cdot & \cdot & \cdot & \cdot \\
\cdot & \cdot & \cdot & \cdot k^{2} / 4 a+k^{2} / 2-(2 n-2)^{2} & k^{2} / 4
\end{array}\right\|
\end{aligned}
$$

it appears that

$$
\frac{a_{2 n}}{a_{0}}=+\frac{4^{n} D_{n}}{k^{2 n}} .
$$

But $a_{2 n} / a_{0}=\left(k^{2}\right)$; hence $D_{n}=\left(k^{2 n+2}\right)$.

This allows the calculation of the successive terms of $a$ as follows. Put $a+k^{2} / 2=c k^{4}+\cdots$; substitute in $D_{2}$ and require that the result shall have the form $\left(k^{6}\right)$. This gives $\left(c k^{4}+\cdots-4\right)\left(c k^{4}+\cdots\right)-k^{4} / 8=\left(k^{6}\right)$, or $-4 c-1 / 8=0$, or $c=-1 / 32$. Next substitute in $D_{3}$ for $a+k^{2} / 2$ the expression $-(1 / 32) k^{4}+c k^{6}+\cdots$ and require that the lowest power of $k^{2}$ which appears shall have a vanishing coefficient. This power is multiplied by $2^{2 \cdot 4^{2}} \cdot c$. Hence $c=0$. The process may be continued; at each stage a new coefficient is found unambiguously, and finally the expression for $a$ in powers of $k$.

15. When $a$ is known the coefficients $a_{2}, a_{4}, \cdots$ are at once determined, but it is preferable to arrange the series for $\operatorname{ce}_{0}(z)$ in powers of $k$, or to put

$$
\mathrm{ce}_{0}(z)=1+b_{2} k^{2}+b_{4} k^{4}+\cdots,
$$

where $b_{2}, b_{1}, \cdots$ are finite trigonometric series. Substitute in the differential equation and give $a$ the value just found in powers of $k$. There results

$$
\begin{gathered}
b_{2}^{\prime \prime} k^{2}+b_{4}^{\prime \prime} k^{4}+\cdots+\left(a+k^{2} / 2\right)\left(1+b_{2} k^{2}+b_{4} k^{4}+\cdots\right) \\
+\left(k^{2} / 2\right)\left(1+b_{2} k^{2}+b_{4} k^{4}+\cdots\right) \cos 2 z=0 .
\end{gathered}
$$

If the coefficients of $k^{2}, k^{4}, \cdots$ are equated to zero, a system of linear differential equations with constant coefficients for $b_{2}, b_{4}, \cdots$ results:

$$
\begin{aligned}
& b_{2}^{\prime \prime}+\frac{1}{2} \cos 2 z=0, \\
& b_{4}^{\prime \prime}-\frac{1}{32}+\frac{1}{2} b_{2} \cos 2 z=0,
\end{aligned}
$$

This simultaneous system may be solved, and the complementary functions must be put equal to zero to obtain $\mathrm{ce}_{0}(z)$. It is found that

$$
\operatorname{ce}_{0}(z)=1+\left(k^{2} / 8\right) \cos 2 z+\left(k^{4} / 2^{9}\right) \cos 4 z+\cdots \text {. }
$$


The value of $a$ is equal to

$$
a=-k^{2} / 2-k^{4} / 2^{5}+0 \cdot k^{6}-\frac{7}{389 \cdot 2} k^{8}+\cdots .
$$

It is necessary to examine the statement made above that the values of the coefficients found for the expansion of $a$ are determined unambiguously. The development of $a$ has been obtained by substitution of the series

$$
c_{1} k^{2}+\cdots+c_{n} k^{2 n}+c_{n+1} k^{2 n+2}+\cdots
$$

for $a$, and by determination of $c_{1}, \cdots, c_{n}$ so that $D_{n}=\left(k^{2 n+2}\right)$. For example if for $a$ is substituted

$$
-k^{2} / 2-(1 / 32) k^{4}+0 \cdot k^{6}+c_{4} k^{8}+\cdots
$$

it is found that

but

$$
D_{1}=\left(k^{4}\right), D_{2}=\left(k^{6}\right), D_{3}=\left(k^{8}\right) ;
$$

$$
D_{4}=\left(a+k^{2} / 2-6^{2}\right) D_{3}-\left(k^{2} / 2\right)^{2} D_{2}=\left(k^{8}\right),
$$

and these equations are true whatever $c_{4}$. If $c_{4}$ can be determined to make $D_{3}=\left(k^{10}\right)$, it follows that $D_{4}=\left(k^{10}\right)$, since $\left(k^{2} / 4\right)^{2} D_{2}=\left(k^{10}\right)$. When $k^{2}=0$, the roots of $D_{n}=0$ are distinct. Then from the principles of algebraic functions, there exists a unique series $c_{1} k^{2}+\cdots$ which expresses that root of the equation $D_{n}=0$ which vanishes with $k^{2}$. The coefficients of this series may be determined seriatim by the conditions that $D_{n}=\left(k^{2}\right), D_{n}=\left(k^{4}\right)$, etc. Applying this theorem to $D_{4}$ it follows that the coefficients of the first three terms are $-1 / 2,-1 / 32,0$. Hence it must be possible to determine $c_{4}$ so as to make $D_{4}=\left(k^{10}\right)$. The subsequent terms in the expansion of $a$ may be similarly determined.

The quantities $a_{2 n}, D_{n}$ are really divisible by higher powers of $k$ than has yet appeared; in fact $a_{2 n}=\left(k^{2 n}\right)$ and $D_{n}=\left(k^{4 n}\right)$. This may be proved from the system of equations for $b_{2}, b_{4}$, etc., which are

$$
b_{2}{ }^{\prime \prime}+c_{n} b_{0}+c_{n-1} b_{2}+c_{n-2} b_{4}+\cdots+c_{2} b_{2 n-4}+\frac{1}{2}(\cos 2 z) b_{2 n-2}=0 \quad(n=1, \cdots)
$$

where $a+k^{2} / 2=c_{2} k^{4}+\cdots, c_{1}=0, b_{0}=1$. It is evident that $b_{2 n}$ is the first unknown which involves $\cos 2 n z$, so that $k^{2 n}$ is the lowest power of $k$ which occurs in the coefficient of $\cos 2 n z$; hence $a_{2 n}=\left(k^{2 n}\right)$. Since

$$
\frac{a_{2 n}}{a_{0}}= \pm \frac{4^{n} D_{n}}{k^{2 n}}
$$

it follows that $D_{2 n}=\left(k^{4 n}\right)$. 
16. The method of determination of $c_{0}(z)$ may be applied to the calculation of all the functions $\mathrm{ce}_{2 n}(z)$. Consider the next even function of period $\pi$, and write

$$
\mathrm{ce}_{2}(z)=a_{0}+a_{2} \cos 2 z+\cdots .
$$

In the system of equations for $a_{0}, a_{2}, \cdots(\S 14)$, put $a_{2}=1$. When $k=0$, $a$ reduces to $2^{2}$ and $a_{0}, a_{4}, a_{6}$ vanish, and so are of the form $\left(k^{2}\right)$. Upon reference to the solution given for $a_{0}, \cdots, a_{2 n}$, it is evident that $D_{n}=\left(k^{2 n}\right)$, $n \geqq 2$. This allows the calculation of $a$ by a method analogous to that used in the case of $\mathrm{ce}_{0}(z)$. From the first equation, $a_{0}=\left(k^{2}\right)$. Substitute in the second and it follows that $a+k^{2} / 2-2^{2}=\left(k^{4}\right)$. Put

$$
a=2^{2}-k^{2} / 2+c k^{4}+\cdots,
$$

and since $D_{2}=\left(k^{4}\right)$ whatever $c$, make $D_{3}=\left(k^{6}\right)$. Then

$$
c=+\frac{5}{3 \cdot 2^{6}} \text {. }
$$

Put

$$
a=2^{2}-\frac{k^{2}}{2}+\frac{5}{3 \cdot 2^{6}} k^{4}+c k^{6}+\cdots,
$$

and make $D_{4}=\left(k^{8}\right)$. Then $c=0$, etc. Hence

$$
a=4-\frac{k^{2}}{2}+\frac{5}{3 \cdot 2^{6}} k^{4}+0 \cdot k^{6}+\cdots
$$

It is needless to repeat the arguments which justify this procedure. The function $\mathrm{ce}_{2}(z)$ is determined by a system of differential equations as was $\mathrm{ce}_{0}(z)$; the result is

$$
\mathrm{ce}_{2}(z)=\cos 2 z+\frac{k^{2}}{2^{5}}(\cos 4 z-2)+\frac{k^{4}}{6 \cdot 2^{10}} \cos 6 z+\cdots .
$$

The results of calculation are here illustrated; $a^{(n)}$ denotes the value of $a$ for $\operatorname{ce}_{n}(z)$ and $a_{(n)}$ the value of $a$ for the $n$th odd function $\operatorname{se}_{n}(z)$ :

$$
\begin{aligned}
& a^{(0)}=-k^{3} / 2-k^{4} / 2^{5}+0 \cdot k^{6}+\cdots, \\
& a^{(1)}=1-\frac{3}{4} k^{2}-\frac{1}{2^{7}} k^{4}+\frac{1}{3 \cdot 2^{12}} k^{6}+\cdots, \\
& a^{(2)}=4-\frac{1}{2} k^{2}+\frac{5}{3 \cdot 2^{6}} k^{4}+0 \cdot k^{6}+\cdots,
\end{aligned}
$$




$$
\begin{aligned}
& a^{(3)}=9-\frac{1}{2} k^{2}-\frac{1}{2^{8}} k^{4}-\frac{1}{2^{12}} k^{6}+\cdots, \\
& a^{(4)}=16-\frac{1}{2} k^{2}-\frac{1}{2^{5} \cdot 15} k^{4}-\frac{11}{2^{9} \cdot 45} k^{6}+\cdots, \\
& a_{(1)}=1-k^{2} / 2^{2}-k^{4} / 2^{7}+\cdots, \\
& \operatorname{ce}_{0}(z)=1+\left(k^{2} / 8\right) \cos 2 z+\left(k^{4} / 2^{9}\right) \cos 4 z+\cdots \text {, } \\
& \operatorname{ce}_{1}(z)=\cos z+\left(k^{2} / 2^{5}\right) \cos 3 z+\left(k^{4} / 2^{10}\right)(\cos 5 z-(1 / 3) \cos 3 z)+\cdots \text {, } \\
& \operatorname{ce}_{2}(z)=\cos 2 z+\left(k^{2} / 2^{5}\right)((2 / 3) \cos 4 z-2)+\frac{k^{4}}{6 \cdot 2^{10}} \cos 6 z+\cdots \text {, } \\
& \operatorname{ce}_{8}(z)=\cos 3 z+\left(k^{2} / 2^{5}\right)\left(-\cos z+\frac{1}{2} \cos 5 z\right) \\
& +\left(k^{4} / 2^{10}\right)(\cos z+(1 / 10) \cos 7 z)+\cdots, \\
& \operatorname{ce}_{4}(z)=\cos 4 z+\left(k^{2} / 2^{5}\right)((2 / 5) \cos 6 z-(2 / 3) \cos 2 z) \\
& +\frac{k^{4}}{2^{10}}\left(\frac{1}{15} \cos 8 z+\frac{88}{45} \cos 2 z+\frac{1}{3}\right)+\cdots \\
& \operatorname{se}_{1}(z)=\sin z+\left(k^{2} / 2^{5}\right) \sin 3 z+\left(k^{4} / 2^{10}\right)((1 / 3) \sin 5 z+\sin 3 z)+\cdots ; *
\end{aligned}
$$

The differential equation

$$
\frac{d^{2} y}{d z^{2}}+\left(a+k^{2} \cos ^{2} z\right) y=0
$$

can be transformed into itself. Put $z=z^{\prime}+\pi / 2$; then

$$
\frac{d^{2} y}{d z^{\prime 2}}+\left(a+k^{2} / 2-\left(k^{2} / 2\right) \cos ^{2} z^{\prime}\right) y=0 \text {. }
$$

This transformation makes possible the calculation of a second function and a second value of $a$, when a first function and a first value of $a$ are given. For example if the transformation is applied to $\mathrm{ce}_{1}(z)$ there results the function $-\operatorname{se}_{1}(z)$, and $a^{(1)}$ is transformed into $a_{(1)}$, etc.; $\operatorname{ce}_{0}(z)$ and $a_{0}$ are exceptional. It is evident that on replacing $z$ by $z^{\prime}+\pi / 2$ and $k^{2}$ by $-k^{2}$, $\operatorname{ce}_{0}(z)$ is transformed into itself. Hence $a_{0}$ is transformed into itself. But this proves that all powers of $k$ in $a_{0}$ after the first term are of the form $4 n$.

* These calculations have been carried further by Dr. Saund, in his doctor dissertation at the University of California. 
17. The assumption has been made, in what precedes, that periodic solutions exist, and that the quantities $a, \cdots, a_{n}, \cdots$ are analytic functions of $k$. It remains to justify these assumptions. In what follows two proofs of the existence of periodic solutions will be given. It may be seen that periodic solutions exist for every value of $k^{2}$. For consider the equations $a_{11}+a_{22}= \pm 2(\& 7)$ in which $\lambda=-k^{2}$, and $h^{2}=-a$. For a given value, $\lambda=\lambda_{0}, a_{11}+a_{22}$ is an integral function of $h$. In accordance with the general theory of Picard, ${ }^{*}$ if one of these equations has not an infinite number of solutions the other one has an infinite number. If the equation $a_{n}+a_{22}=2$ has an infinite number of solutions there exists for the value $\lambda=\lambda_{0}$ an infinite number of functions having period $\pi$. If $a_{11}+a_{22}=-2$ has an infinite number of solutions, there exists an infinite number of functions $f(x)$ such that $f(x+\pi)=-f(x)$, or $f(x+2 \pi)=f(x)$. Hence there exists for all values of $\lambda$ an infinite number of functions having the period $\pi$ or $2 \pi$. This theorem is not sufficient to prove the existence, for all values of $k^{2}$, of the functions $\mathrm{ce}_{n}(z), \mathrm{se}_{n}(z)$ for which formal developments have just been obtained.

A determination of all periodic solutions of the differential equation will now be given in the form of a proof that the series for $\operatorname{ce}_{n}(z)$, $\operatorname{se}_{n}(z)$ and $a^{(n)}, a_{(n)}$ converge for all values of $k^{2}$. Write the system of equations for $a_{0}, a_{2}, \cdots$ as follows. Put

$$
\frac{k^{2}}{4\left(a+k^{2} / 2-(2 n)^{2}\right)}=x_{n},
$$

and $a+k^{2} / 2=v$; then the equations beginning at the third are

$$
\begin{aligned}
& x_{2} c_{1}+c_{2}+x_{2} c_{3}=0, \\
& x_{3} c_{2}+c_{3}+x_{3} c_{4}=0,
\end{aligned}
$$

where $c_{n}=a_{2 n}$. This is a system of the same form as that previously treated $(\S 4)$. A solution such that $c_{n} \rightarrow 0$ was found to be given by

$c_{1}: c_{2}: \cdots c_{n}: \cdots=\left(x_{2}\right):-x_{2}\left(x_{3}\right): \cdots:(-1)^{n} x_{2} \cdots x_{n+1}\left(x_{n+2}\right): \cdots$

It is supposed that $v \neq(2 n)^{2}$ so that $x_{n} \neq \infty$. Substitute the values of $c_{1}, c_{2}$ in the first and second equations ( $\$ 14)$ and they become

$$
v a_{0}+\left(k^{2} / 4\right)\left|\begin{array}{ccccc}
1 & x_{2} & \cdot & \cdot & \cdot \\
\cdot & \cdot & \cdot & \cdot & \cdot \\
\cdot & \cdot & \cdot & \cdot & \cdot
\end{array}\right|=0,
$$

* Picard, Traité d'A nalyse, vol. 3, p. 346. 


$$
\left(k^{2} / 2\right) a_{0}+\left(v-2^{2}\right)\left|\begin{array}{cccc}
1 & x_{2} & \cdot & \cdot \\
\cdot & \cdot & \cdot & \cdot \\
\cdot & \cdot & \cdot & \cdot
\end{array}\right|-x_{2}\left(k^{2} / 4\right)\left|\begin{array}{cccc}
1 & x_{3} & \cdot & \cdot \\
\cdot & \cdot & \cdot & \cdot \\
\cdot & \cdot & \cdot & \cdot
\end{array}\right|=0 .
$$

Eliminating $a_{0}$ there results

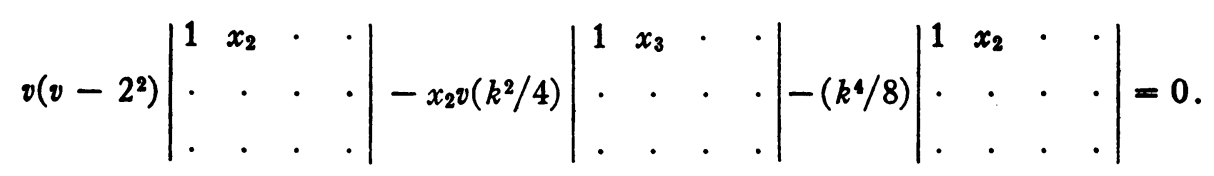

This may be written in the form

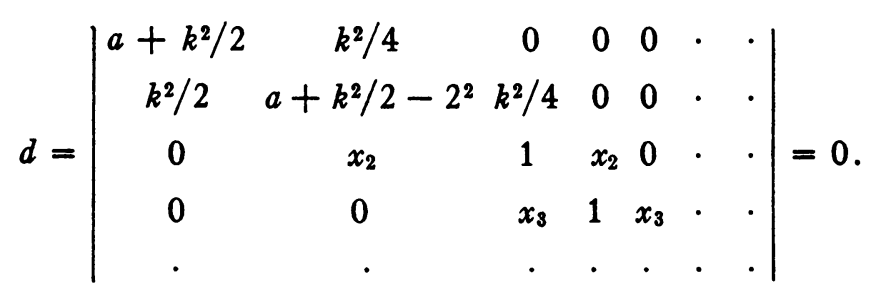

If $D_{n}=\left(a+k^{2} / 2-4^{2}\right)\left(a+k^{2} / 2-6^{2}\right) \cdots\left(a+k^{2} / 2-(2 n-2)^{2}\right) d_{n}$, then $d_{n} \rightarrow d$ as $n \rightarrow \infty$. This follows since

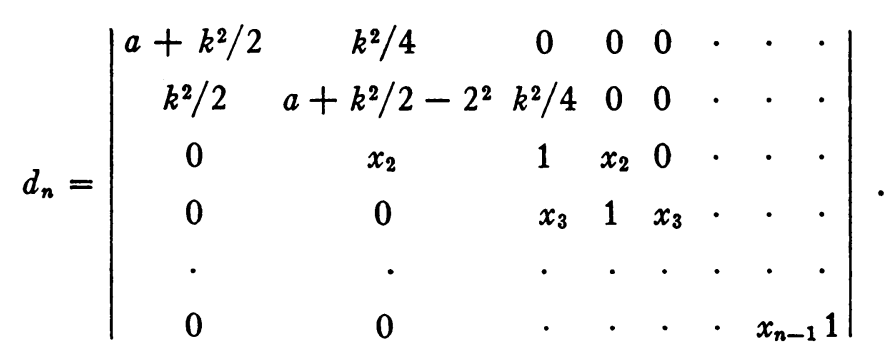

This determinant is convergent as may be seen on applying the test for convergence used in $\S 2$. Hence $d_{n} \rightarrow d, n \rightarrow \infty$. The equation for $v$ given above may evidently be written $v=P_{1}\left(v, k^{2}\right)$ where every term in $P_{1}\left(v, k^{2}\right)$ is divisible by either $v^{2}$ or $k^{2}$; hence $v=P\left(k^{2}\right)$ and it is clear that $P\left(k^{2}\right)=\left(k^{4}\right)$. The value of $a$ corresponding to $v$ is therefore $-k^{2} / 2+c k^{4}+\cdots$, the terms of which have been calculated above ( $\S 14)$. It will be seen that the equation $d=0$ has an infinite number of real roots of which this value of $a$ is the first, and they reduce when $k=0$ to $0,2^{2}, 4^{2}, \ldots$ This is proved in the following way. If the determinant $d$ is expanded in terms of the row containing $x_{n}$ the equation $d=0$ may be written in the form

$$
v-(2 n)^{2}=P_{1}\left(v-(2 n)^{2}, \quad k^{2}\right),
$$


where every term in $P_{1}\left(v-(2 n)^{2}, k^{2}\right)$ is divisible by $\left(v-(2 n)^{2}\right)$ or $k^{2}$. Accordingly $v-(2 n)^{2}=P\left(k^{2}\right)$, or

$$
a=(2 n)^{2}-k^{2} / 2+P\left(k^{2}\right) .
$$

These values of $a$ may all be calculated from the equations $D_{n}=0$ in the manner carried out in $\S 14$.

In this way the roots which tend to $(2 n)^{2}$ as $k \rightarrow 0$ are found. It may be proved that there are no others, or more precisely, no root of the equation $d=0$ tends to a limit, $l \neq(2 n)^{2}$, and no root increases indefinitely as $k \rightarrow 0$. The first statement is true since $a$ and $k^{2}$ satisfy the equation $a_{11}+a_{22}-2=0$. If a root of this equation in $a$ is bounded as $k \rightarrow 0$ it must have a limit. This limit is a root of the equation $a_{11}+a_{22}-2=0$ in which $k$ has been put equal to zero. Further a root of the equation $d=0$ cannot $\rightarrow \infty$ when $k \rightarrow 0$. If $v \rightarrow \infty$ then $|v|$ takes the value $(2 p+1)^{2}$ infinitely often, and

$$
\left|v-(2 n)^{2}\right| \geqq|| v\left|-(2 n)^{2}\right|=\left|(2 p+1)^{2}-(2 n)^{2}\right| .
$$

The least value of this quantity for a given $p$ is furnished by $n=p$, so that $\left|v-(2 n)^{2}\right| \geqq 4 p+1$; hence $x_{n} \rightarrow 0$ for these values of $|v|$. But the equation $d=0$ may be written

$$
v(v-2)^{2}\left|\begin{array}{ccccccc}
1 & x_{0} & 0 & 0 & . & . & . \\
2 x_{1} & 1 & x_{1} & 0 & . & . & . \\
0 & x_{2} & 1 & x_{2} & . & . & . \\
. & . & . & . & . & . & .
\end{array}\right|=0,
$$

and cannot be satisfied if $|v|=2 p+1, p \rightarrow \infty$. Hence as $k \rightarrow 0$ the roots of $d=0 \rightarrow 0,2^{2}, 4^{2}, \cdots$. The same reasoning shows that when $k \rightarrow k_{0} \neq 0$ no root of the equation $d=0 \rightarrow \infty$.

If a value of $a$ satisfying $d=0$ is obtained the values of the coefficients $a_{2}, a_{4}, \cdots$ are given in two different forms in what precedes, viz.

$$
a_{2 n}=(-1)^{n} \frac{4^{n} D_{n}}{k^{2 n}} \quad\left(n=0,1, \cdots, D_{0}=1\right),
$$

and $a_{2 n}=c_{n} / c_{0}$ where

$$
\begin{aligned}
& c_{n}=(-1)^{n} x_{2}, \cdots, x_{n+1}\left(x_{n+2}\right) \quad(n=1,2, \cdots), \\
& c_{0}=\frac{k^{2}}{4\left(a+k^{2} / 2\right)} a_{2}=-x_{0}\left(x_{2}\right) .
\end{aligned}
$$

The series $1+a_{2} \cos 2 z+\cdots$ is convergent because the ratio 


$$
r_{n}=\frac{a_{2 n}}{a_{2 n-2}}=-\frac{x_{2} \cdots x_{n+1}\left(x_{n+2}\right)}{x_{2} \cdots x_{n}\left(x_{n+1}\right)}=-\frac{x_{n+1}\left(x_{n+2}\right)}{\left(x_{n+1}\right)}, n>1 ;
$$

and since $\left(x_{n}\right) \rightarrow 1$ as $n \rightarrow \infty$ it follows that

$$
r_{n}=-\frac{k^{2}}{4\left\{a+k^{2} / 2-(2 n+2)^{2}\right\}}+\epsilon .
$$

Hence the series $1+a_{2} \cos 2 z+\cdots$ converges for all values of $z$.

By a similar reasoning the existence of the odd functions as well as those of period $2 \pi$ may be proved.

It has been assumed in the preceding analysis that $a+k^{2} / 2 \neq(2 n)^{2}$. If $a+k^{2} / 2=(2 n)^{2}$ the proof of convergence may be effected by beginning at the $n$th equation for $a_{0}, a_{2}, \cdots$ and expressing the solution of the system

$$
\begin{aligned}
& x_{n+1} c_{n}+c_{n+1}+x_{n+1} c_{n+2}=0, \\
& x_{n+2} c_{n+1}+c_{n+2}+x_{n+2} c_{n+3}=0,
\end{aligned}
$$

in the same manner as before.

The functions denoted $\mathrm{ce}_{n}(z), \mathrm{se}_{n}(z)$ are obtained from the expressions just given for the periodic solution by dividing by the coefficient of cos nz or of $\sin n z$, so that these terms have coefficients equal to unity. This division is possible if $k$ is sufficiently small since the divisors are $\neq 0$.

Finally the existence of periodic solutions has been proved and expressions for all of them for all values of $k^{2}$ have been determined.

18. The series obtained for the quantities $a$ and the periodic functions converge if $k$ is sufficiently small, but the radius of convergence has not been found. Consider the values of $a$ which belong to $c_{2 n}(z)$; it may be proved that the singular points of $a$ are those values of $k$ for which two or more values of $a$ coincide. For if $k_{0}$ is the singular value of $k$ then when $k \rightarrow k_{0}$, it was proved above that $a \rightarrow 1$, a finite value; and if the values of $a$ corresponding to $k=k_{0}$ are distinct, each of the values of $a$ is by the theory of implicit functions a series of integral powers of $k-k_{0}$. But the value $k_{0}$ would not be singular if the values of $a$ are so expressed; hence the values of $a$ are not distinct if $k=k_{0}$.

If $f_{n}(z), f_{m}(z)$ are two periodic functions corresponding to two values of $a$ which become equal, then $f_{n}(z)=\rho f_{m}(z), \rho$ independent of $z$, when $k=k_{0}$. Since by the theory of integral equations

$$
\int_{-\pi}^{\pi} f_{n}(z) f_{m}(z) d z=0
$$


it follows that if $k=k_{0}$,

$$
\int_{-\pi}^{\pi} f_{n}^{2}(z) d z=0
$$

The value of this integral may be expressed in terms of $a_{2}, a_{4}, \cdots$ giving

$$
\int_{-\pi}^{\pi} f_{n}^{2}(z) d z=\left(1+\frac{1}{2} a_{2}^{2}+\frac{1}{2} a_{4}^{2}+\cdots\right) 2 \pi .
$$

Hence if for $k=k_{0}$ two values of $a$ become equal, and if in

$$
a_{2 n}=(-1)^{n} \frac{4^{n} D_{n}}{k^{2 n}},
$$

the value with which they coincide is substituted, then

$$
1+\frac{1}{2} a_{2}^{2}+\frac{1}{2} a_{4}^{2}+\cdots=0 .
$$

It follows that the singular values $k_{0}{ }^{2}$ are not real. To prove this it is necessary to prove first that if $k^{2}$ is real the values of $a$ are real. Suppose that for a real value of $k^{2}, a$ has the value $a^{\prime}+i a^{\prime \prime}$ and $y$ has the value $y_{1}+i y_{2}$. Then

$$
y_{1}^{\prime \prime}+i y_{2}^{\prime \prime}+\left(a^{\prime}+i a^{\prime \prime}+k_{2} \cos ^{2} z\right)\left(y_{1}+i y_{2}\right)=0
$$

or

$$
\begin{aligned}
& y_{1}^{\prime \prime}+\left(a^{\prime}+k^{2} \cos ^{2} z\right) y_{1}-a^{\prime \prime} y_{2}=0 \\
& y_{2}^{\prime \prime}+\left(a^{\prime}+k^{2} \cos ^{2} z\right) y_{2}+a^{\prime} y_{1}=0
\end{aligned}
$$

It follows that

$$
\frac{d}{d z}\left(y_{2} y_{1}^{\prime}-y_{1} y_{2}^{\prime}\right)=a^{\prime \prime}\left(y_{1}^{2}+y_{2}^{2}\right)
$$

and integrating from 0 to $\pi$ that

$$
0=a^{\prime \prime} \int_{0}^{\pi}\left(y_{1}^{2}+y_{2}^{2}\right) d z
$$

since $y_{1}$ and $y_{2}$ have the period $\pi$. Hence $a^{\prime \prime}=0$ or $a$ is real. If $a$ and $k^{2}$ are real, $a_{2 n}$ is real so that

$$
1+\frac{1}{2} a_{2}^{2}+\frac{1}{2} a_{4}^{2}+\cdots \neq 0 .
$$

Hence $k_{0}^{2}$ is not real if $k_{0}$ is a singular value. Since the singular values of $k$ correspond to equal values of $a$ the equation $d=0$ cannot have a multiple root if $k^{2}$ is real.

A numerical estimate of the values of $k^{2}$ and $a$ which are singular may be found from the polynomials $D_{n}$ by finding the values of $k^{2}$ for which 
the equation $D_{n}=0$ has coincident roots. It appears that approximately $k^{2}=5.8 i, a=2.1-2.9 i$ for the singular point nearest zero.

19. The equation of the elliptic cylinder cannot have two periodic solutions of period $\pi$ or $\pi i$ for the same values of $a$ and $k^{2}$. If $\mu=0$ two solutions are given by the formulas

$$
\begin{aligned}
& f(z)=\frac{1}{\phi(0)} \sum_{-\infty}^{+\infty} \phi(n) J_{n}\left(\lambda e^{-s}\right) J_{n}\left(\lambda e^{z}\right), \\
& \psi(z)=\frac{1}{\phi(0)} \sum_{-\infty}^{+\infty} \phi(n) J_{n}\left(\lambda e^{-s}\right) K_{n}\left(\lambda e^{z}\right) .
\end{aligned}
$$

The second solution is distinct from the first, since

$$
K_{n}(x)=c(\log x) J_{n}(x)+P_{n}(x),
$$

where $c$ is not zero and $P_{n}(x)$ is a power series in $x$. This solution does not vanish identically since this would require $\phi(n)=0$ for every $n$. Since every solution is a linear combination of $f(z)$ and $\psi(z)$, and since every solution is periodic if two independent solutions are periodic, $\psi(z)$ must be periodic. But

$$
\psi(z)=c(\log \lambda) e^{z} f(z)+\sum_{-\infty}^{+\infty} \phi(n) J_{n}\left(\lambda e^{-z}\right) P_{n}\left(\lambda e^{z}\right),
$$

which is not periodic. Hence the equation of the elliptic cylinder never has two independent periodic solutions.

An incidental result of the theorem that two values $a_{(2 n)}, a_{(2 m)}$ cannot coincide when $k^{2}$ is real is that $a_{(2 m)}>a_{(2 n)}$ if $m>n$. For if $a_{(2 m)}<a_{(2 n)}$ for any value of $k$, letting $k^{2}$ diminish to zero, there must for some value of $k^{2}$ be found the equality $a_{(2 m)}=a_{(2 n)}$, since when $k^{2}=0, a_{(2 m)}=2 m^{2}$ and $a_{(2 n)}=(2 n)^{2}$. But $a_{(2 m)} \neq a_{(2 n)}$ for any real value of $k^{2}$. Hence $a_{(2 m)}>a_{(2 n)}$ if $m>n$.

It may further be proved that if $k^{2}$ is real the value of $d a / d k^{2}$ is negative. First it may be proved that $d a / d k^{2} \neq 0$ for any real value of $k^{2}$. For suppose $d a / d k^{2}=0$ and let $w=d y_{1} / d k^{2}$ where $y_{1}$ is a periodic solution. Then if $y_{2}$ is an independent solution it may be supposed that $y_{2}=z y_{1}+y_{3}$ where $y_{3}$ is periodic. The function $w$ must satisfy the equation

$$
\frac{d^{2} w}{d z^{2}}+\left(a+k^{2} \cos z\right) w=-y_{1} \cos ^{2} z,
$$


and the solution of this equation is known to be

where

$$
w=A y_{2}+\frac{y_{2}}{c} \int-\left(\cos ^{2} z\right) y_{1}^{2} d z+B y_{1}-\frac{y_{1}}{c} \int-\left(\cos ^{2} z\right) y_{1} y_{2} d z,
$$

If

$$
c=y_{1} \frac{d y_{2}}{d z}-y_{2} \frac{d y_{1}}{d z} \neq 0 .
$$

$$
-\left(\cos ^{2} z\right) y_{1}^{2}=A_{0}+A_{1} \cos 2 z+\cdots
$$

it is found that

$$
w=\frac{1}{2} A_{0} z^{2} y_{1}+z F(z)+G(z),
$$

where $F(z)$ and $G(z)$ are periodic functions of $z$. Hence if $w$ is periodic, $A_{0}=0$. But

hence

$$
A_{0}=-\frac{1}{2 \pi} \int_{0}^{2 \pi}\left(\cos ^{2} z\right) y_{1}^{2} d z
$$

$$
\int_{0}^{2 \pi}\left(\cos ^{2} z\right) y_{1}^{2} d z=0
$$

which is impossible if $k^{2}$ is real. Hence $d a / d k^{2} \neq 0$. The values of $d a / d k^{2}$ when $k=0$ are known however from the series for $a$, viz., $-k^{2} / 2-k^{4} / 32$, $1-3 k^{2} / 4-\cdots$, and are seen to be $-1 / 2,-1 / 3,-1 / 2, \cdots$, the values being $-1 / 2$ after the second. Hence $d a / d k^{2}<0$ for all real values of $k^{2}$, which was to be proved.

Universtry OF CaLtrornia, Berkruey, Calif. 